Distribution Category: Atomic, Molecular, and Chemical Physics (UC-411)

\author{
ARGONNE NATIONAL LABORATORY \\ 9700 South Cass Avenue \\ Argonne, Illinois 60439
}

ANL/APS/LS-260

\title{
MEASUREMENT OF GAS BREMSSTRAHLUNG FROM THE INSERTION DEVICE BEAMLINES OF THE ADVANCED PHOTON SOURCE
}

M. Pisharody and P. K. Job

Experimental Facilities Division Advanced Photon Source

\section{S. Magill, J. Proudfoot, and R. Stanek}

High Energy Physics Division

March 1997

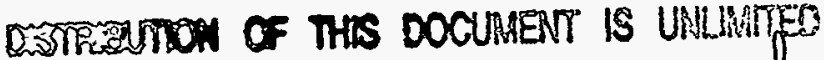

work sponsored by

U.S. DEPARTMENT OF ENERGY

Office of Energy Research

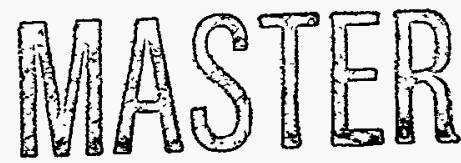




\section{DISCLAIMER}

Portions of this document may be illegible in electronic image products. Images are produced from the best available original document. 


\section{Acknowledgements}

The authors wish to thank Mark Keeffe for his assistance in designing the hardware for the lead glass calorimeter mount, Bill McHargue for providing the attenuator circuit module and associated electronics, and Brian Rodricks for his help with the data acquisition (DAQ) components and software. We are also thankful to Ed Semones for his help in the residual gas analysis (RGA) effort, and Don Jankowski for his technical assistance. Special acknowledgements to Harold Moe for his valuable suggestions during the course of the experiment and analysis. Finally, the authors are grateful to the members of the APS Collaborative Access Teams (CATs) belonging to Sectors 10 (MR CAT), 12 (BESSRC CAT), and 13 (GSE CARS CAT) for providing the beamlines in order to conduct the experiment.

This work is supported by U.S. Department of Energy, BES-Material Sciences, under contract no. W-31-109-ENG-38.

iii 


\section{Summary}

High energy electron storage rings generate energetic bremsstrahlung photons through radiative interaction of the electrons (or positrons) with the residual gas molecules inside the storage ring. The resulting radiation exits at an average emittance angle of $\left(\frac{m_{0} c^{2}}{E}\right)$ radian with respect to the electron beam path, where $m_{0} c^{2}$ is the rest mass of the electron and $\mathrm{E}$ its kinetic energy. Thus, at straight sections of the storage rings, moving electrons will produce a narrow and intense monodirectional photon beam. At synchrotron radiation facilities, where beamlines are channeled out of the storage ring, a continuous gas bremsstrahlung spectrum, with a maximum energy of the electron beam, will be present.

There are a number of compelling reasons that a measurement of the bremsstrahlung characteristics be conducted at the Advanced Photon Source (APS) storage ring. Although the number of residual gas molecules present in the storage ring at typical nTorr vacuum is low, because of the long straight paths of the electrons in the storage ring at APS, significant production of bremsstrahlung will be produced. This may pose a radiation hazard. It is then imperative that personnel be shielded from dose rates due to this radiation. There are not many measurements available for gas bremsstrahlung, especially for higher electron beam energies. The quantitative estimates of gas bremsstrahlung from storage rings as evaluated by Monte Carlo codes also have several uncertainties. They are in general calculated for air at atmospheric pressure, the results of which are then extrapolated to typical storage ring vacuum values (of the order of $10^{-9}$ Torr). Realistically, the actual pressure profile can vary inside the narrow vacuum chamber. Also, the actual chemical composition of the residual gas inside the storage ring is generally different from that of air. 
The $7 \mathrm{GeV}$ stored electron beam energy and the long $(5 \mathrm{~m})$ insertion device (ID) straight sections with long straight paths, approximately $5 \mathrm{~m}$ on either side of the IDs, provide us with a unique opportunity to carry out the gas bremsstrahlung measurements. The first optical enclosures (FOE) of the ID beamlines are suitable to perform such measurements. The ID is kept fully open to minimize the synchrotron radiation background. The bremsstrahlung beam emerges as a narrow cone along the beamline into the FOE with a characteristic opening angle of $73 \mu \mathrm{rad}$, making it a few millimeters in diameter downstream of the ID in the FOE. Such a narrow intense bremsstrahlung beam can only be measured accurately using a high energy electromagnetic calorimeter that possess good resolution and a fast time response. Lead glass has the desired properties and a segmented array of this material with the right dimensions will practically contain the entire bulk of electromagnetic shower generated by the incident bremsstrahlung beam. Such a calorimeter has enabled us to accurately measure the bremsstrahlung energy spectrum and rate.

This report describes the measurements of the gas bremsstrahlung characteristics downstream of ID beamlines of the APS storage ring. Using a $5 \times 5$ array of lead glass detectors with photomultiplier readouts and an associated data acquisition system, measurements of the gas bremsstrahlung spectrum and intensity were conducted as a function of storage ring parameters, such as beam current and vacuum. Total integrated gas bremsstrahlung energy radiated in normalized units of $\mathrm{GeV} / \mathrm{sec} / \mathrm{nT} / \mathrm{mA}$ was then evaluated, and the resulting maximum dose equivalent was estimated using existing conversion factors. The experiment was repeated at various ID beamlines as different experimental runs.

The report details the experimental setup, data acquisition system, data-taking procedures, analysis of the data, and the results of the measurements. 


\section{Contents}

Acknowledgements

Summary $\quad$ v

1 Introduction 1

1.1 Bremsstrahlung from Electron Storage Rings . . . . . . . . . . . 1

1.2 Previous Measurements . . . . . . . . . . . . . . . . 3

1.3 Bremsstrahlung Dose Rate . . . . . . . . . . . . . . . . 3

2 The Experimental Setup and Data Acquisition System 7

2.1 Electromagnetic Shower . . . . . . . . . . . . . . 7

2.2 Lead Glass Calorimeter . . . . . . . . . . . . . . . . 8

2.3 Data Acquisition System . . . . . . . . . . . . . . . 12

3 Experimental Procedures and Data Collection 15

3.1 Calorimeter Alignment . . . . . . . . . . . . . . . 15

3.2 Data Collection Procedure . . . . . . . . . . . . 16

4 Results and Analysis $\quad 19$

4.1 The Raw ADC Bremsstrahlung Spectrum . . . . . . . . . . . . 19

4.2 Calibration Procedure ....................... 20

4.3 Data Analysis . . . . . . . . . . . . . . . . 28

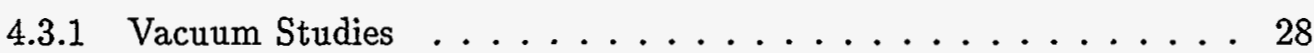

4.3.2 Multiple Bremsstrahlung Events ............. 30 
4.3.3 The Residual Gas Analysis . . . . . . . . . . . . . . 33

4.3.4 The Measured Bremsstrahlung Energy Spectrum . . . . . . . . 35

4.4 Systematic Errors . . . . . . . . . . . . . . . . . . 36

4.4 .1 Dead-Time Correction . . . . . . . . . . . . . 39

4.4 .2 Longitudinal Shower Leakage Correction . . . . . . . . . . . . 39

4.4.3 Low Energy or Threshold Correction . . . . . . . . . . . . . . . 42

4.5 Corrected Results for Bremsstrahlung Energy . . . . . . . . . . . 45

4.6 Bremsstrahlung Dose Estimations . . . . . . . . . . . . . . . 49

5 Conclusions $\quad 55$

$\begin{array}{ll}\text { Bibliography } & 57\end{array}$ 


\section{List of Tables}

1 Lead glass detector calibration constants and resolutions as obtained from fitting corresponding $A D C$ bremsstrahlung spectrum by Equation $6 . \mathrm{C}_{13}$ is approximately twice higher compared to the rest because of a factor of two less attenuation pre-set in this ADC channel to compensate for half of its signal used for the trigger. . . . . . . . . . . . . . . . . . . . . . .

2 Summary of the RGA studies. . . . . . . . . . . . . . 35

3 Observed dead-time-corrected bremsstrahlung energy originating from the 15.38 meter particle beam straight path (not corrected for other systematic errors). . . . . . . . . . . . . . . . 38

4 Low energy correction evaluated for a few typical beam currents. . . . . . 45

5 Corrected bremsstrahlung energy results from the 15.38 meter particle beam straight path. . . . . . . . . . . . . . 46

6 Normalized maximum dose equivalent values corresponding to the bremsstrahlung energy radiated from the $15.38 \mathrm{~m}$ of particle beam straight path. Only one typical value per run is shown. . . . . . . . . 54 


\section{List of Figures}

1 The RE1 lead glass electromagnetic calorimeter. . . . . . . . . 9

2 Pedestal-subtracted ADC distribution for various PMT high voltages, using a pulsed LED source attached to the front of a typical lead glass detector. As an example, the inset shows details such as $\angle A D C>$ and $\sigma_{A D C}$ of the hatched distribution for 2100 volts. The photoelectron yield in this case is approximately $1220 \ldots \ldots \ldots \ldots \ldots \ldots$

3 (a) Calculated photoelectron yields $\left(\mathrm{N}_{p e}\right)$ for various $\mathrm{PMT}$ high voltages, obtained using Equation (4). (b) Calculated gains ( $G$ ) as a function of PMT high voltages as determined by Equation (5). . . . . . . . 11

4 Schematic of the RE1 data acquisition system. . . . . . . . . 13

5 Side view of the RE1 lead glass electromagnetic calorimeter that shows a $0.5 \mathrm{X}_{0}$ copper plate shielding the synchrotron background from bending magnets. . . . . . . . . . . . . . . . . . 16

6 Typical uncorrected raw ADC pedestal-subtracted spectra from central 3 $\times 3$ lead glass blocks of the calorimeter. The center block (\#13) contains $\sim 92 \%$ of the energy deposited. Lateral energy spread is visible in all the surrounding blocks. The storage ring contained electrons at $60 \mathrm{~mA}$ during

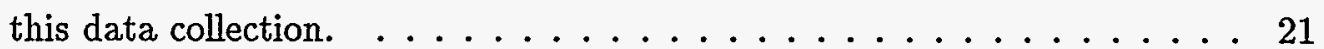

7 (a) An expected ADC distribution generated by monte carlo simulation with a calibration of $250 \mathrm{ADC} / \mathrm{GeV}$ and a resolution of $10 \% / \sqrt{ } E_{\gamma}$. (b) The endpoint of (a) with the fit described in equation (6). The fit results show that we can correctly measure the calibration constant $\mathrm{C}$ and the

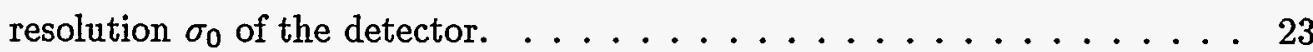


8 The summed ADC spectrum of all nine lead glass block detectors of the $\mathrm{RE} 1$ calorimeter. Additional entries after the cutoff are most likely to be from multi-bremsstrahlung events as discussed in the text. The inset shows the fit to the end-point region of the spectrum using Equation 6 , which yields values for the calibration constant and resolution of the

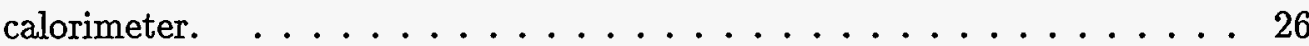

9 The uncorrected bremsstrahlung energy spectrum. Calibration constant $\mathrm{C}_{\text {eff }}$ obtained from the fit procedure has been used to convert the ADC distribution shown in Figure 7. The cutoff, smeared by the finite resolution of the calorimeter, can be seen at around $6.77 \mathrm{GeV} . \ldots . \ldots 27$

10 Layout of a sector $\operatorname{ID}(\mathrm{N})$ of the APS storage ring. The photon beamline from $\mathrm{ID}(\mathrm{N})$ traverses also through the sector $\mathrm{ID}(\mathrm{N}+1)$. Locations of various ion pumps (IP) and ion gauges (IG) that are in sector $N$ and $N+1$ are shown. The photon beamline of $\operatorname{ID}(\mathrm{N})$ separates from the storage ring after the IP2 pump location of sector $(\mathrm{N}+1)$. The figure is not to scale. . 29

11 The typical vacuum value profiles (in nTorr) from sector 12 (IP4(12), IP5(12), IG(12), and IP6(12) ) and sector 13 (IP1(13) and IP2(13)), shown for a period of 24 hrs. The storage ring had been operating with electron beam during the hours of the day shown here. . . . . . . . 31

12 The vacuum readings (in nTorr) from IP2(13), IP5(12), and IG(12) for a typical data collection duration time of 16 minutes. No significant variations are observed in the vacuum values during the data collection interval.

13 Simulated bremsstrahlung spectra. The solid line is the $\frac{1}{E}$ spectrum with detector resolution smearing. The spectrum represented by the dotted line is the superposition of $\frac{1}{E}$ as well as a $1 \% \frac{1}{E^{2}}$ entries of the total number of events. The extra events in the dotted spectrum beyond the cutoff are visible. 
14 The gas bremsstrahlung energy spectra, corrected for dead-time losses, for typical beam currents from the electron beam runs \#1 and \#2 in sector 12 ID (Figures $a$ and $b$ ) and from the positron beam runs \#3 in sector $10 \mathrm{ID}$ and \#4 in sector $13 \mathrm{ID}$ (Figures $\mathrm{c}$ and $\mathrm{d}$ ). The $\mathrm{y}$-axis represents the photon production rate $\langle N\rangle$ normalized to the vacuum. The lowest and highest beam currents are marked by the respective spectra, and the currents for the intermediate plots are given on the side in that order. . . 37

15 Energy spectra uncorrected and corrected for dead-time from the electron run \#2 in sector $12 \mathrm{ID}$, for $60 \mathrm{~mA}$ (a) and $100 \mathrm{~mA}$ (b) beam currents. . 40

16 EGS4 Monte Carlo results. (a) Incoming bremsstrahlung energy spectrum. (b) The shower leakage energy profile at the back side of the RE1 calorimeter. (c) Scatter plot between (a) and (b) showing the correlation.

17 Typical dead-time corrected bremsstrahlung spectra with the y-axis in units of $\mathrm{E}_{\gamma} \times$ Rate $\langle\mathrm{N}\rangle$. The $1 / \mathrm{E}_{\gamma}$ profiles in Figure 12 now yield flat distributions. ...................... 43

18 Dead-time corrected bremsstrahlung spectrum with the $y$-axis in units of $\mathrm{E}_{\gamma} \times$ Rate $\langle\mathrm{N}\rangle$ fit with a straight line yielding the fit parameters $\mathrm{A}$ and $\mathrm{B}$ (inset). . . . . . . . . . . . . . . . .

19 Bremsstrahlung energy, normalized to the beam current, as a function of the storage ring vacuum for the experimental runs conducted at sectors 10-ID and 12-ID. The slopes of the fitted straight lines give the gas bremsstrahlung energy rate, the value of which is given at the top. The $y$-intercepts represent the non-gas bremsstrahlung energy rate from these beamlines. . . . . . . . . . . . . . . . . 47

20 Data from the run conducted at sector 13-ID along with the other three data sets shown in Figure 18. The gas bremsstrahlung energy rate from 13-ID is approximately a factor of four higher compared to those from sectors $10-\mathrm{ID}$ and $12-\mathrm{ID}$, consistent with a variation in the effective $\mathrm{Z}$ of the storage ring residual gas due to vacuum leak. . . . . . . . . 48 
21 The fluence-to-dose-equivalent conversion factors (Sv.cm ${ }^{2}$ ) vs depth curves for varying incident photon energies $\left(E_{\gamma}^{i n c} \mathrm{GeV}\right)$. The results are obtained from calculations conducted by D.W.O. Rogers [19]. . . . . . 51

22 The calculated largest fluence-to-dose-equivalent conversion factors within the $30 \mathrm{~cm}$ ICRU4 tissue thickness as a function of incident bremsstrahlung energy. ........................ 53 


\section{Chapter 1}

\section{Introduction}

\subsection{Bremsstrahlung from Electron Storage Rings}

Bremsstrahlung or "breaking radiation" is emitted by a charged particle as it decelerates due to the inelastic radiative interaction with the Coulomb force field of atomic nuclei and electrons of the medium it traverses through. The charged particle is deflected in this process and imparts a significant fraction of its kinetic energy to the emitted photon. The energy spectrum of the emitted photon ranges from zero to the initial energy of the interacting charged particle. Both for analysis of experiments and for an understanding of the details of this process, the pioneering work of Bethe is of great importance [1]. In his analytical treatment of bremsstrahlung theory, he obtained the photon spectrum in a very simple form, which was later extended to relativistic energies by Heitler [2]. The differential atomic cross section for the bremsstrahlung emission is proportional to the square of the atomic number $\left(\mathrm{Z}^{2}\right)$ of the material and, for a nonrelativistic charged particle, depends on the inverse square of the mass of the particle. However, as the velocity of the particle approaches relativistic values, its mass is of decreasing importance. Thus bremsstrahlung generation by charged particles other than electrons is not easily detected unless they have relativistic velocities.

From the experimental stand point, electrons have been the most important source 
of bremsstrahlung. The differential cross section $d \sigma$ for the emission of a photon in the energy range between $E_{\gamma}$ and $E_{\gamma}+d E_{\gamma}$, by an incident electron of kinetic energy $E$, in the material of nuclei charge Ze can be expressed as:

$$
d \sigma=\sigma_{0} B\left(Z, E_{\gamma} / E\right) Z^{2} \frac{\left(E+m_{0} c^{2}\right)}{E} \frac{d E_{\gamma}}{E_{\gamma}} \quad\left(\mathrm{cm}^{2} / \text { nucleus }\right)
$$

where $\sigma_{0}=\frac{1}{137} \frac{e^{4}}{m_{0}^{2} c^{4}}, m_{0} c^{2}$ being the rest mass of the electron, and $B$ is a coefficient that is a slowly varying function of $\mathrm{Z}$ and $\mathrm{E}_{\gamma} / \mathrm{E}$. The numerical evaluation of $\mathrm{B}$ as a function of $\mathrm{Z}$ and $\mathrm{E}_{\gamma} / \mathrm{E}$ has been done by Heitler [2] and has been verified for particle energies $\leq 500 \mathrm{MeV}$. Evaluation of this function at higher energies has not been done. Although a comprehensive theory of bremsstrahlung at all particle energies does not exist as of yet, several equations that could be used for different electron energies and various materials are available $[3,4]$.

In an electron storage ring, beams of electrons (or positrons) circulate for periods of the order of several hours between refills. The particles are guided around a roughly circular closed orbit by a collection of bending and focusing magnets. The ring is kept at high vacuum to minimize bremsstrahlung energy loss due to interaction of the particles with the residual gas molecules in the ring. Pressures of $10^{-8}$ to $10^{-9}$ Torr are typical. The synchrotron radiation energy loss of the circulating particles due to bending magnetic fields and insertion devices is made up by a radio-frequency (rf) accelerating system.

The Advanced Photon Source (APS) at Argonne National Laboratory consists of a $200 \mathrm{MeV}$ electron linac, a positron converter target, a $450 \mathrm{MeV}$ positron linac, a positron accumulator ring, a $7 \mathrm{GeV}$ booster synchrotron, and a $7 \mathrm{GeV}$ electron/positron storage ring along with a number of experimental photon beamlines utilizing the synchrotron radiation [5]. Bremsstrahlung is produced in the APS storage ring when the circulating beam scatters from the storage-ring components and/or from the residual gas molecules in the vacuum [6]. Interactions of the beam with the storage-ring components generally occur when it deviates from the ideal closed orbit or during injection and beam dumps. Scattering from the residual gas molecules in the ring vacuum occurs continuously during operation, which generates the gas bremsstrahlung. Gas bremsstrahlung becomes very important for large storage rings with long straight beam paths on either side of the 
insertion device (ID) straight sections, as the contribution from each interaction adds up to produce a narrow monodirectional bremsstrahlung beam. This beam travels down the beamline, along with the synchrotron radiation, with a characteristic emission angle of $73 \mu \mathrm{rad}[7]$. Because the storage ring beam straight paths at the APS are 15.38 meters long (in the line of sight of its beamline), gas bremsstrahlung in the beamlines is significant. Bremsstrahlung can develop into an electromagnetic shower within any beamline component it encounters. In addition, it can also generate photoneutrons from targets of high-Z materials [8] like photon beam stops and collimators.

\subsection{Previous Measurements}

There are not many measurements available for gas bremsstrahlung, especially at higher electron energies. A few gas bremsstrahlung experimental measurements have been performed in the storage rings at KEK [9] in Japan and Frascati $[10,11,12]$ in Italy. These measurements were done at lower energies compared to the $7 \mathrm{GeV}$ particle beam energy of the APS storage ring. Monte Carlo electromagnetic shower codes [13] have been used to quantitatively estimate gas bremsstrahlung from storage rings. These codes assume air at atmospheric pressure as the particle's traveling medium. The results were then linearly extrapolated to vacuum values of a typical storage ring. But the vacuum profiles along the length of the straight paths in the storage ring are difficult to measure. Also, the chemical composition of the residual gas molecules along the particle trajectory in the storage ring vacuum chamber is different from that of air and may even change during the course of storage ring operation. Furthermore, in order to accumulate a large number of events for good statistics in a Monte Carlo simulation of particle transport in air, several days of CPU time would be required. In previous studies [14], the discrepancy between the reported measurements and the Monte Carlo simulations is at least a factor of 3 .

\subsection{Bremsstrahlung Dose Rate}

The total beam-integrated gas bremsstrahlung dose rate $\mathrm{D}(\mathrm{Sv} / \mathrm{hr}$ ) from the straight particle trajectory in the vacuum chamber of the storage ring at a distance $\mathrm{L}$ from the 
end of the straight path is usually approximated by a semiempirical equation [15]:

$$
D=\frac{f N l}{\Pi \alpha^{2} X_{0} L(L+l)}
$$

where:

$\mathrm{f}=$ an effective flux-to-dose conversion factor for bremsstrahlung photons $\left(3.0 \times 10^{-6} \mathrm{~Gy} / \mathrm{hr} . f l u x\right)$,

$\alpha=$ ratio of positron rest mass to its kinetic energy (0.511/7000.),

$\mathrm{N}=$ the number of charged particles for a given beam current (for $300 \mathrm{~mA}=1.872 \times 10^{18} / \mathrm{sec}$ ),

$\mathrm{X}_{0}=$ radiation length of air $\left(\right.$ at $10^{-9}$ Torr $\left.=2.34 \times 10^{16} \mathrm{~cm}\right)$,

$l=$ effective length of the straight path available for the particle (for the APS $=1538 \mathrm{~cm}$ ), and

$\mathrm{L}=$ distance from the end of the straight path to the detector (at the APS, typically $=2460 \mathrm{~cm}$ ).

Using Equation 2, the total beam-integrated gas bremsstrahlung dose rate, for an APS straight beam path, is approximately estimated to be $2.2 \mathrm{~Sv} / \mathrm{hr}$ at $300 \mathrm{~mA}$. Therefore an actual experimental measurement of this dose rate is very important from the point of view of the radiation protection of the personnel and radiation shielding. Measurements, especially as a function of the storage ring parameters, will be of great interest.

The first optical enclosures (FOE) or white beam stations of the ID beamlines at the APS, which are adequately shielded, are suitable for measurements of gas bremsstrahlung characteristics. Because we are only interested in the bremsstrahlung dose, the ID is kept fully open in order to minimize the synchrotron radiation background. However, there is a small contribution from the synchrotron radiation produced by the bending magnets which travels along the straight path of the particle, and arrives at the detector. This synchrotron energy spectrum ranges from 0 to $500 \mathrm{KeV}$ with a critical energy of 20 $\mathrm{KeV}$. A thin shield can be used to stop this remaining residual synchrotron radiation. The measurement of the narrow monodirectional gas bremsstrahlung photons can be conducted by means of a sensitive electromagnetic calorimeter. Such a calorimeter should possess good resolution at energies around a few $\mathrm{GeV}$ in order to measure the photon energy spectrum as accurately as possible. The number of bremsstrahlung photons produced per second in the APS storage ring segment of length $15.38 \mathrm{~m}$ is approximately 
estimated as $1.2 \times 10^{5}$ photons $/ \mathrm{sec}$. The calorimeter should also have a fast time response to cope with such bremsstrahlung rates.

The lead glass blocks with a radiation length of about $2.6 \mathrm{~cm}$, containing approximately $51 \% \mathrm{PbO}$, is an ideal material for the desired calorimeter. The energy resolution of this device is typically about $5-10 \% / \sqrt{E_{\gamma}}$. The characteristic response time of lead glass is considered fast and is typically about $20 \mathrm{nsec}$. Lead glass having a depth of about $20 \mathrm{X}_{0}$ will contain practically $100 \%$ of the electromagnetic shower longitudinally. The transverse development of electromagnetic showers in different materials scales fairly accurately with the Moliere radius. The Moliere radius of lead glass is about $3 \mathrm{~cm}$, which means that an electromagnetic cascade can be completely contained transversely in a $30 \mathrm{~cm}$ diameter block (only about 5 Moliere radii of material is necessary to laterally contain an electromagnetic shower).

A radiation experiment collaboration was formed between the Advanced Photon Source (APS) Experimental Facilities Division (XFD) and the High Energy Physics (HEP) division at Argonne National Laboratory (ANL) to build a detector and associated data acquisition system and to experimentally measure the gas bremsstrahlung energy spectrum and rate in an APS ID beamline straight section. The experiment, known as Radiation Experiment 1 (RE1), has been performed at beamlines 10-ID, 12-ID, and 13-ID of the APS, using an electromagnetic calorimeter made of 25 lead glass detectors, each $6.3 \mathrm{~cm} \times 6.3 \mathrm{~cm} \times 35 \mathrm{~cm}$, stacked into a $5 \times 5$ array. Experimental data for varying storage ring parameters have been logged into a data acquisition system for analysis. The remaining part of this report contains detailed information regarding the experimental setup, data acquisition system, measurement procedures, analysis of the data acquired, and discussion of the results. 


\section{Chapter 2}

\section{The Experimental Setup and Data Acquisition System}

\subsection{Electromagnetic Shower}

Photons entering a medium will undergo one or more of five interaction processes depending on their energy and the nature of the medium traversed. They are:

(1) Atomic photoelectric efFect,

(2) Compton or Incoherent scattering,

(3) Pair production (in electron or nuclear field),

(4) Rayleigh or Coherent scattering, and

(5) Photonuclear interactions.

The first three of these are the most important from the point of view of transfer of photon energy to matter. Rayleigh scattering is a redirection of the photon with no energy loss. The photonuclear interaction takes place for photon energies above a few $\mathrm{MeV}$ resulting usually in the production of neutrons. The relative contributions from the Compton effect, photoelectric effect, and pair production depend both on the photon energy and the atomic number $\mathrm{Z}$ of the medium of interaction. The photoelectric effect is dominant at lower photon energies, the Compton effect at medium energies, and pair production at high energies. The exact energy ranges in which each of these processes 
occurs vary with the atomic number $\mathrm{Z}$ of the medium. These three processes transfer the photon energy to the media via electrons, which, with repeated radiative and collision interactions, generate a multiplicative electromagnetic shower.

\subsection{Lead Glass Calorimeter}

Electromagnetic calorimeters are detectors that measure the energy of incident electrons or photons by converting the energy of the electromagnetic shower into measurable light. A homogeneous electromagnetic calorimeter is made entirely from an active material, and the signal is read out from the entire volume of the calorimeter. Thus, in order to accurately measure the energy of the photon from gas bremsstrahlung, the calorimeter should be homogeneous and must contain the bulk of the electromagnetic shower.

The RE1 detector is shown in Figure 1. It consists of 25 lead glass blocks tightly stacked into a $5 \times 5$ array. Lead glass material is composed of $50.8 \% \mathrm{PbO}, 41 \% \mathrm{SiO}_{2}$, and $7 \% \mathrm{~K}_{2} \mathrm{O}$ by weight, and has a radiation length $\left(\mathrm{X}_{0}\right)$ of $2.6 \mathrm{~cm}$. Each lead glass block used has dimensions of $6.3 \mathrm{~cm} \times 6.3 \mathrm{~cm} \times 35 \mathrm{~cm}\left(13.5 \mathrm{X}_{0}\right)$ and is enclosed in a metal light-tight can with a photomultiplier tube (PMT) and base fastened to the end. An LED can be attached to the center of the front of each detector for gain and stability studies. The PMTs are powered by a 36 channel LeCroy $1454 \mathrm{HV}$ system and are read out through coaxial cables into two LeCroy 16-input CAMAC 4300B FERAs (fast encoding and readout $A D C s$ ). During data acquisition, the incident photon beam is aligned on the center of the central lead glass block of the $5 \times 5$ matrix (see Figure 1). The lateral dimension of the entire bulk of the detector is sufficient to contain the electromagnetic shower in the transverse direction and will contain about $96 \%$ of the shower longitudinally (approximately $20 \mathrm{X}_{0}$ of any material is required to fully contain an electromagnetic shower in the longitudinal direction).

Linearity of the ADCs was checked using the FERA on-board DAC with the results better than $0.1 \%$. Using a pulsed LED source, the photoelectron yield and PMT gain were measured as a function of the high voltage by the following method.

The ADC distribution of the pulsed LED source follows Poisson statistics, the mean of which $(\angle \mathrm{ADC}\rangle)$ changes with applied high voltage. An example of such a distribution 


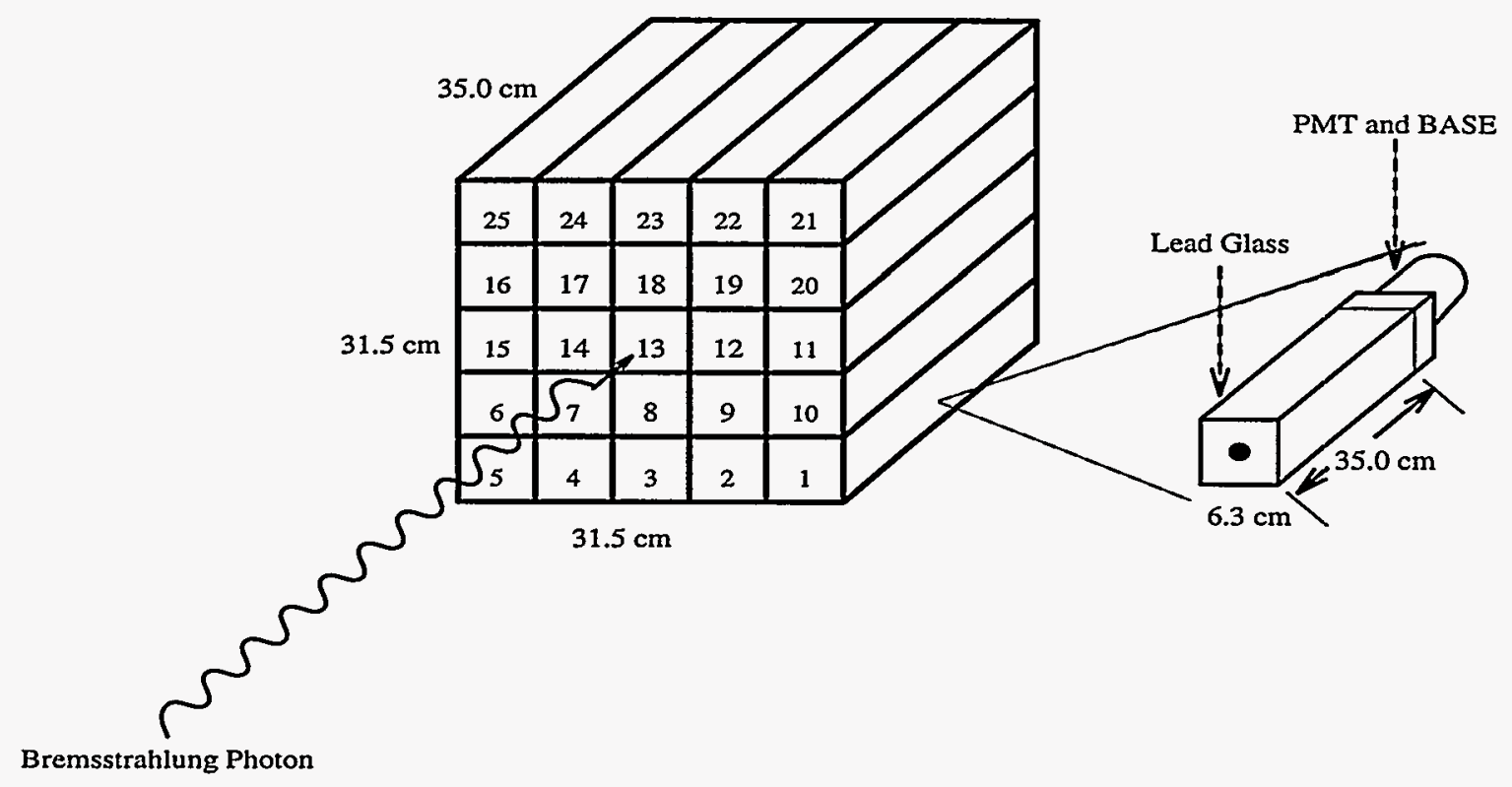

Figure 1: The RE1 lead glass electromagnetic calorimeter.

for a typical lead glass detector is given in Figure 2. Using the mean value <ADC> and width $\sigma_{A D C}$ of the distribution, the number of photoelectrons $\mathrm{N}_{p e}$ is approximated as:

$$
N_{p e}=\left(\frac{\left\langle A D C>-P_{A D C}\right.}{\sigma_{A D C}}\right)^{2},
$$

where $\mathrm{P}_{A D C}$ is the mean $\mathrm{ADC}$ pedestal. The gain is then determined from the relation:

$$
G=\frac{\langle A D C>}{N_{p e}} \text {. }
$$

Figure 3 shows the variation of the photoelectron yield and gain as a function of the high voltage appled to the lead glass detector given in Figure 2.

The operating point of the phototubes depends on both the number of photoelectrons expected and the region of linear operation. The phototubes are XP2017 and the minimum operating voltage for linear response is 1700 volts. We estimated the expected photoelectron yield by using the endpoint of the bremsstrahlung spectrum which was obtained in an early measurement made with a single lead glass block. By matching the pulse height for the end point of the bremsstrahlung with the mean pulse height from an LED at identical operating conditions, the number of photoelectrons at $7 \mathrm{GeV}$ was found to be approximately 2700 . 


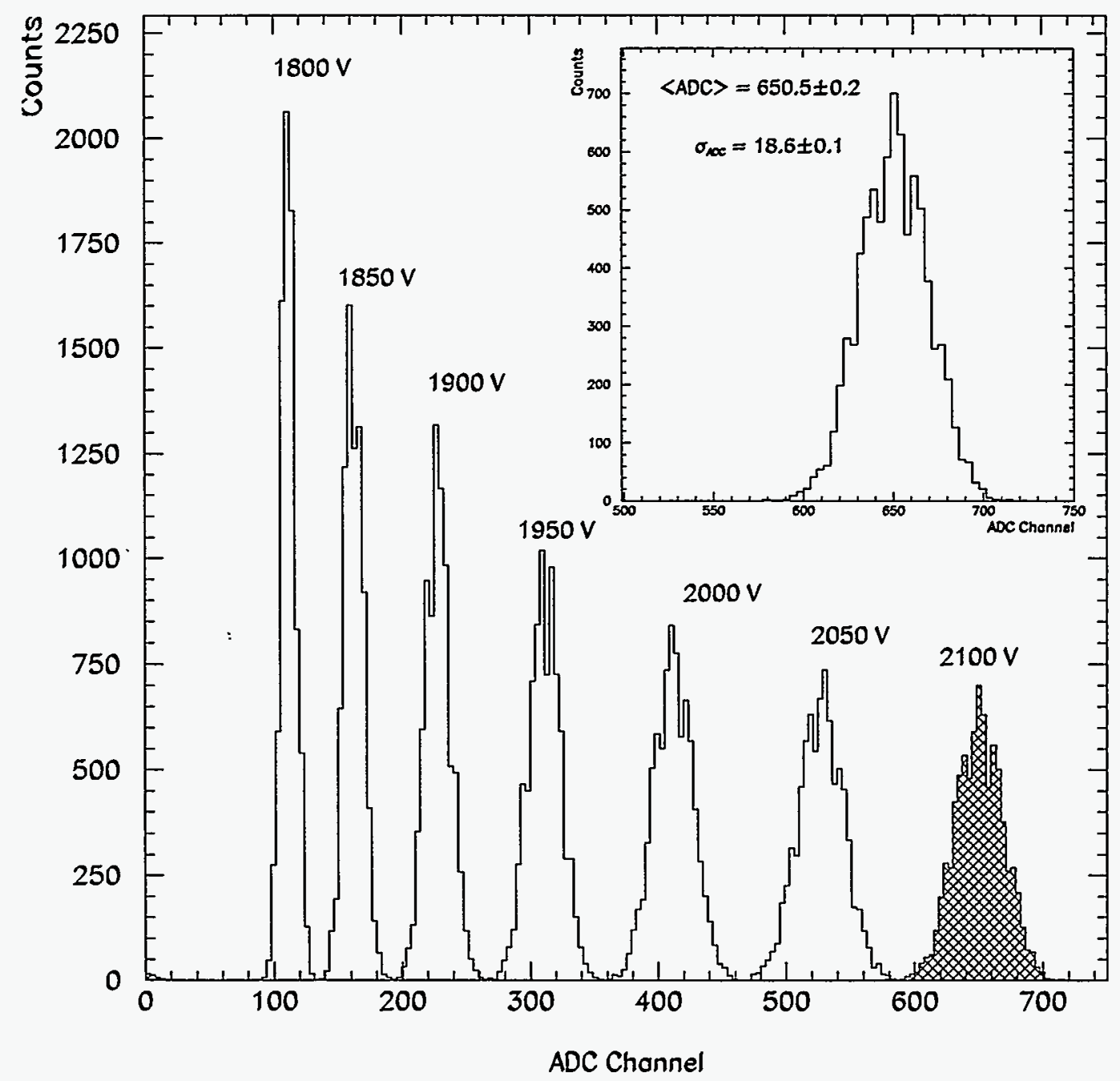

Figure 2: Pedestal-subtracted ADC distribution for various PMT high voltages, using a pulsed LED source attached to the front of a typical lead glass detector. As an example, the inset shows details such as $\angle A D C>$ and $\sigma_{A D C}$ of the hatched distribution for 2100 volts. The photoelectron yield in this case is approximately 1220 . 

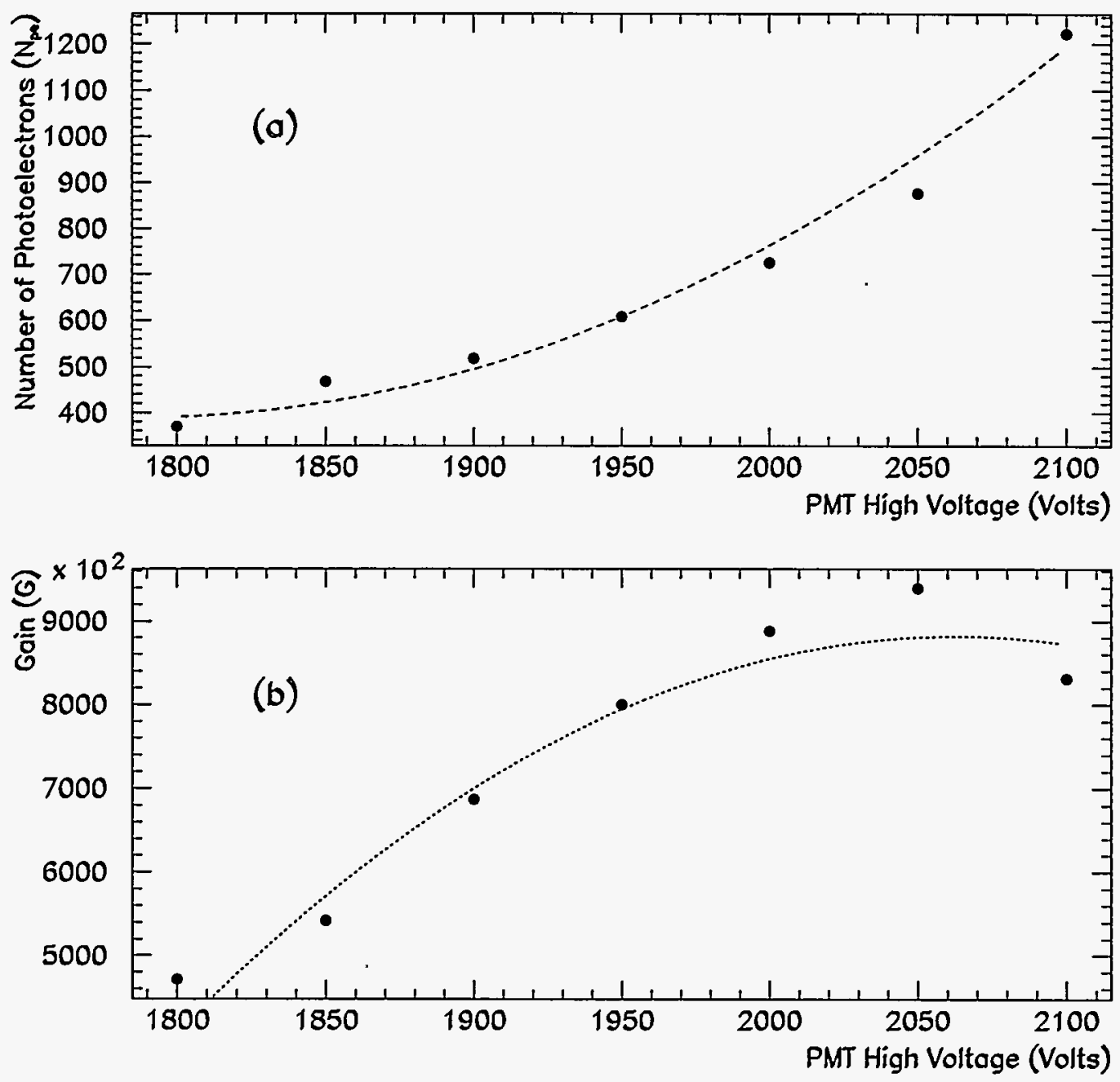

Figure 3: (a) Calculated photoelectron yields $\left(\mathrm{N}_{p e}\right)$ for various PMT high voltages, obtained using Equation (4). (b) Calculated gains (G) as a function of PMT high voltages as determined by Equation (5). 
The initial high voltages are then chosen in such a way that each of the tubes operate in their linear region with approximately similar gains. Typical HVs ranged anywhere between $1800 \mathrm{~V}$ to $2000 \mathrm{~V}$. Final gains of all the tubes were set using the end point of the bremsstrahlung spectrum during the actual experimental measurement. This procedure is described in Section 4.2.

The calorimeter is mounted on a platform that slides on a pair of rails. A step motor is used to power the sliding mechanism. The detector volume can thus be moved beam-left or beam-right using this arrangement. This sliding surface with the detector attached is then placed on a table that can be manually raised and lowered. These lateral and vertical movements thus enable us to scan each lead glass block with the bremsstrahlung beam.

\subsection{Data Acquisition System}

A schematic of the data acquisition system (DAQ) is shown in Figure 4. Signals from individual phototubes of the calorimeter are read out into the ADCs through a passive attenuator resistor array. The ADCs are each of 11 bit dynamic range and have a constant short conversion time of $8.5 \mu \mathrm{sec}$. Control signals from the LeCroy 4301 FERA Driver are daisy chained through the two ADC modules. The attenuators prevent the ADCs from overflowing (the gain of the PMTs at our operating voltage is roughly $10^{6}$, and with about 400 photoelectrons/ $\mathrm{GeV}$, the upper end of the spectrum will easily overflow unless the PMT outputs are attenuated). The attenuation factor is chosen so as to make use of the full dynamic range of the 11 bit ADCs with as little overflow as possible. The attenuation on each channel has been set to approximately $25 \mathrm{~dB}$, except for the central phototube which splits half of its current to the trigger circuit as shown in Figure 4. To compensate for half of the signal that is split, the attenuation on this channel has been adjusted by a factor of two less than the remaining channels.

The trigger is used both to generate a gate to the ADCs upon a real energy deposit, as well as to exclude low energy synchrotron radiation, which is orders of magnitude more intense than the gas bremsstrahlung. A $150 \mathrm{MeV}$ threshold is set using a LeCroy $4608 \mathrm{C}$ octal discriminator. Bremsstrahlung energy lost in this cutoff region is then corrected 


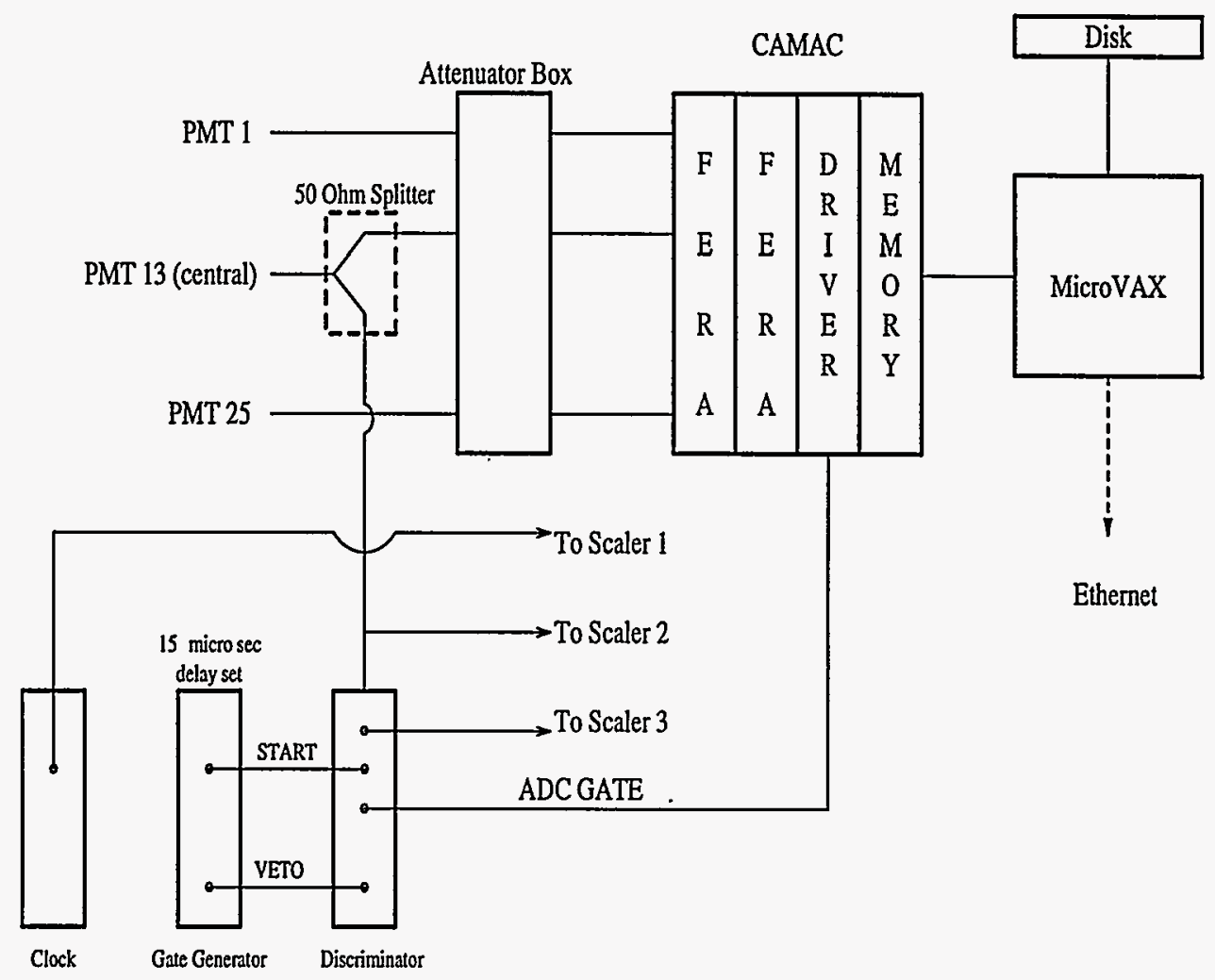

Figure 4: Schematic of the RE1 data acquisition system.

for in the final analysis using a fit and extrapolation procedure. Also, a delay has been introduced to signals from all the channels relative to the central detector. A delay of about $30 \mathrm{~ns}$ is maintained between the leading edge of the gate and the input signals. The typical phototube event signal measures about $20 \mathrm{~ns}$ in width with a long tail. The $\mathrm{ADC}$ gate width is set at $350 \mathrm{~ns}$ to ensure proper charge collection from such a long-tailed pulse.

Since the transfer of acquired data to a computer typically takes many milliseconds per event, the digitized data from the $\mathrm{ADC}$ is stored into a LeCroy $430216 \mathrm{~K}$ memory unit for subsequent slow read out by the computer. Transfer of data from the ADCs to the memory occurs after each gate and proceeds at $100 \mathrm{~ns}$ per word. This eliminates significant dead-time corrections that will have to be applied otherwise. Once the memory unit buffer is full, an inhibit is generated that prevents further readouts of the ADCs 
by the driver. The LAM signals the computer that the buffer is to be readout. After reading out the full memory unit, the computer resets the memory address of the unit and clears the ADC inhibit for the next batch of events. A MicroVax computer is used to control the CAMAC modules and to store the data.

The digitization time of FERA is about $8.5 \mu \mathrm{sec}$ and readout time by the driver into the memory module is about $2 \mu \mathrm{sec}$. This implies an approximate dead time of $11 \mu \mathrm{sec}$ between accepted triggers. Thus the ADCs will convert and the memory will store all events triggered up to a rate of about $90 \mathrm{KHz}(1 / 11 \mu \mathrm{sec})$. Any trigger rate above this will have to be dead-time corrected in the analysis. By introducing a known delay time $\tau$ between ADC gates, the measured rate $N_{M}$ can be corrected for dead time by using the relation:

$$
N_{R}=\frac{N_{M}}{1-N_{M} \tau}
$$

where $N_{R}$ is the dead-time corrected actual trigger rate. The delay $\tau$ is set at $15 \mu \mathrm{sec}$ close to the ADC dead time of $11 \mu \mathrm{sec}$.

Apart from the ADC data, 3 scalers are also written out into the data stream as shown in Figure 4. They include:

1. $\mathrm{S} 1$ - a pulser/clock running at a fixed frequency $(10 \mathrm{KHz})$ for the duration of each data set collection, which is used to establish the rate of triggered events in the data analysis.

2. S2 - a number representing the raw, dead-timeless triggered events.

3. S3 - a number representing the accepted triggers within the dead time limit.

The scalers giving the raw and accepted triggers are also displayed through Quad Counters (EG\&G Ortec Model 974) during the data acquisition for visual reference. 


\section{Chapter 3}

\section{Experimental Procedures and Data Collection}

\subsection{Calorimeter Alignment}

The APS storage ring insertion device (ID) sector 12 was used for the experiment during the electron beam phase, and ID sectors 10 and 13 were utilized for the positron beam. The lead glass detector setup is placed approximately 3 feet away from the ID front end, inside the experimental station. The ID is kept in full open position to eliminate synchrotron radiation. Residual synchrotron background from the upstream and downstream bending magnets of the sector is screened from entering the detector by placing a $0.5 \mathrm{X}_{0}$ copper plate on the calorimeter front face as shown in Figure 5. The shielding plate is $6 \mathrm{~mm}$ thick and has dimensions of $35.4 \mathrm{~cm} \times 35.4 \mathrm{~cm}$. The bremsstrahlung energy loss in this shield has been found to be insignificant ( $1 \%$ of incident energy) from Monte Carlo calculations.

The calorimeter is first aligned along the beam path by means of a few quick beam imaging steps using radiation-sensitive paper. In this procedure, the central lead glass block of the calorimeter is visually aligned approximately along the beam path. A radiation-sensitive paper is then fixed at the center of the shielding copper plate, marking the position of the central lead glass block of the calorimeter. After securing the station, 


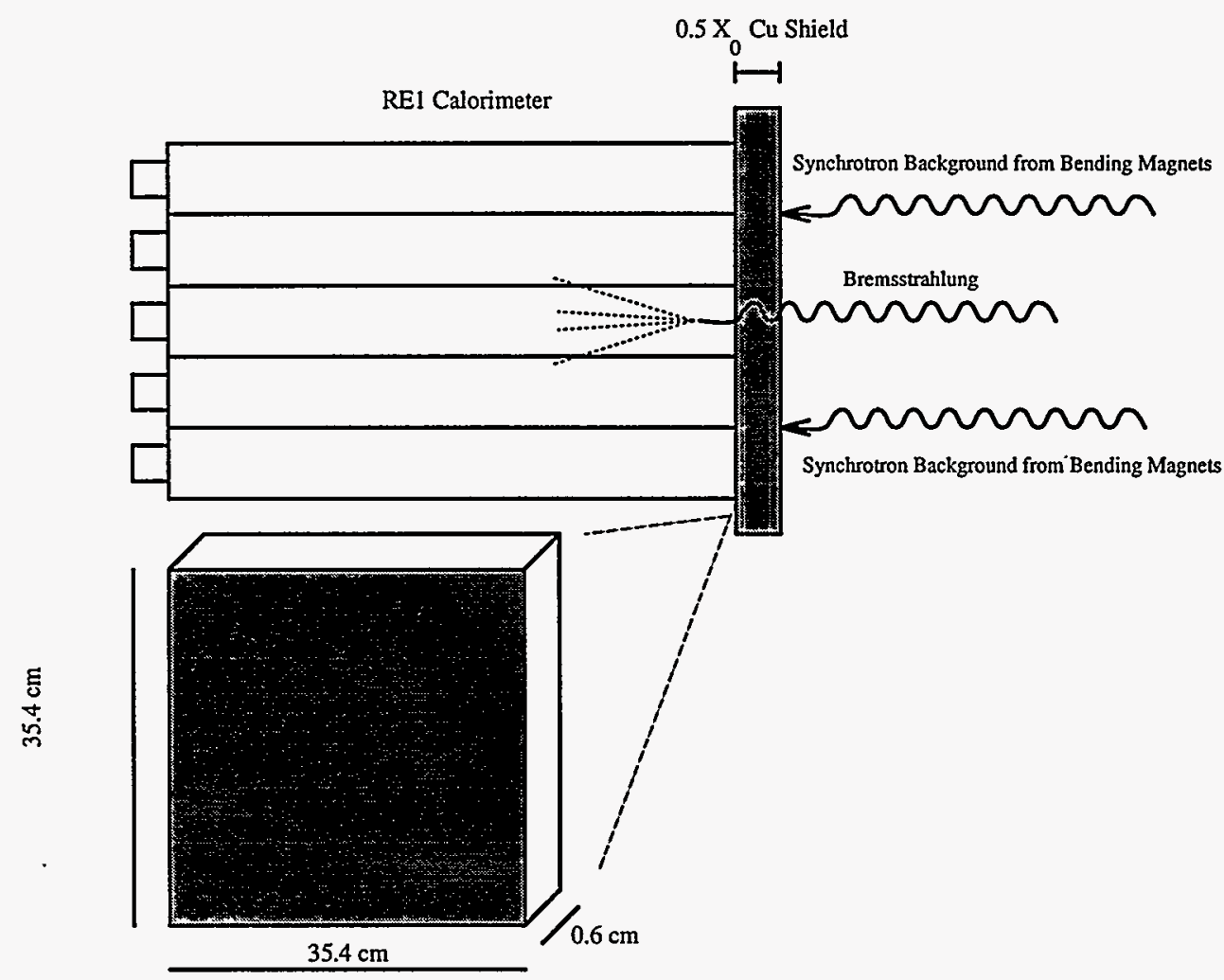

Figure 5: Side view of the RE1 lead glass electromagnetic calorimeter that shows a $0.5 \mathrm{X}_{0}$ copper plate shielding the synchrotron background from bending magnets.

the ID beamline shutter is opened for a few seconds allowing the residual synchrotron beam (along with the bremsstrahlung radiation) from the bending magnets to strike the radiation-sensitive paper. A clear burn spot is generated on the paper, marking the incoming beam position. The central lead glass block is positioned along this photon beam. The beam can now be centered on any of the lead glass blocks of the calorimeter by making use of the calibrated step motor and the crank handle of the setup table.

\subsection{Data Collection Procedure}

During the data-taking phase of the experiment, the beam is aligned to strike the center of the central lead glass detector of the calorimeter. The station is then searched and 
secured and the shutter is opened allowing the bremsstrahlung and residual synchrotron photons to emerge through the front end into the aligned calorimeter. Each photon generates an electromagnetic shower in the calorimeter volume, which will be counted as an event by the data acquisition system. Those events satisfying the pre-set discriminator threshold, trigger the gate and the corresponding ADC digitized outputs are written out to the MicroVaxII hard disk, which also controls the CAMAC electronic crate. The scalers along with $25 \mathrm{ADC}$ channel signals are stored on an event per event basis. Different runs are performed corresponding to different storage ring beam currents. Vacuum values from relevant ion pumps (IPs) and ion gauges (IGs) in the straight section are also recorded for the time period of each run. The day, start time, and end time of the run, and rate of event as seen in the quad scaler are also recorded for each run. The ADC pedestal runs are also conducted from time to time using an external gate from a random pulser to trigger. This information is later used to pedestal-subtract the experimental data written out. During a pedestal run, the station shutter is kept closed to prevent the photon beam from interacting with the calorimeter. 


\section{Chapter 4}

\section{Results and Analysis}

The Advanced Photon Source (APS) stored electrons in the storage ring from the time it became operational until July of 1996. Positrons have been stored from then on, starting from August of 1996. The data in this report come from both of these periods. Apart from understanding the gas bremsstrahlung characteristics in general, it has been our intention to investigate further the differences, if any, in the production of bremsstrahlung as the transition from a stored beam of electrons to that of positrons takes place. Approximately $2.5 \mathrm{~Gb}$ worth of data have been analyzed for results from the combined electron and positron runs. All the data files are kept and analyzed in a Sun unix workstation. Most of the analyses are carried out using the PAW software from the CERN Computing Library, CERNLIB Version 95a.

\subsection{The Raw ADC Bremsstrahlung Spectrum}

During the preliminary stages of analyzing the data, it has been observed that electromagnetic showers from the photon events are fully contained laterally within the central $3 \times 3$ lead glass block volume (about 6 Moliere radii) of the calorimeter (Figure 1). No energy deposition is found in the outermost 16 lead glass blocks of the calorimeter. Therefore, only signals from the central $3 \times 3$ blocks are utilized for all subsequent analysis purposes. As shown in Figure 1, these 9 detectors are numbered 17, 18, 19, 14, 13, $12,7,8,9$, with 13 being the central block. 
Typical pedestal-subtracted raw ADC spectra from each of the nine lead glass blocks are shown in Figure 6. Lead glass block \#13, the center of which is aligned with the photon beam path, shows the bremsstrahlung spectrum with the characteristic $1 / \mathrm{E}$ dependence and the cutoff that defines the end point corresponding to the maximum energy of the particle in the storage ring. The smearing of the knee at the cutoff is due to the finite resolution of the lead glass detector. The very low energy end of the spectrum shows the effect of the discriminator threshold setup. Approximately $92 \%$ of the incoming photon energy is found to be deposited in the central block itself. The rest is distributed over the other eight surrounding blocks.

\subsection{Calibration Procedure}

As discussed in Chapter 1, the PMT gains have so far been set only by a pulsed LED source. The bremsstrahlung beam itself is used to fine-tune the gains more accurately before the data acquisition. The procedure involves scanning each lead glass blocks of the calorimeter by the bremsstrahlung beam. The beam is made to fall at the center of each lead glass block using the mechanism that moves the calorimeter. Individual outputs are then displayed through a $\mathrm{qVt}$ multichannel analyzer module. The observed bremsstrahlung distribution drops at the maximum particle energy of $7 \mathrm{GeV}$, thus defining a sharp endpoint for the spectrum. This well-defined endpoint is used to fine-tune the PMT gains. By scanning the beam from block to block, the individual PMT high voltages are adjusted within their respective operating ranges, so as to fix the endpoint of the bremsstrahlung distribution from each detector approximately at the same qVt channel. Data for the purpose of calibration were written out during the scanning procedure for approximately identical beam currents.

The end point of the measured bremsstrahlung spectrum is also used to determine the absolute calibration of the calorimeter. This technique has been used successfully to determine calorimeter calibration constants [16] and to measure e-p collider luminosity at the HERA electron-proton collider at Hamburg. The raw ADC spectrum as seen in Figure $6(\# 13)$ is a convolution of the bremsstrahlung photon energy distribution with the Bethe-Heitler cross section, $\frac{d \sigma}{d E_{\gamma}} \propto \frac{1}{E_{\gamma}}$, and the Gaussian smearing due to the 


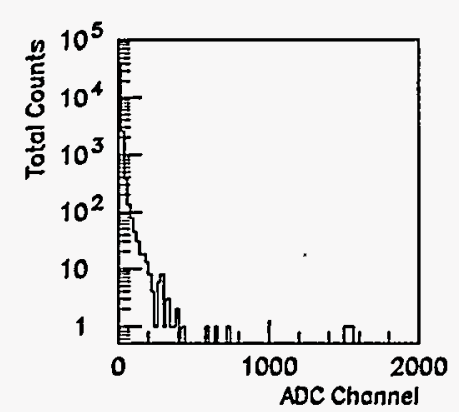

\#17

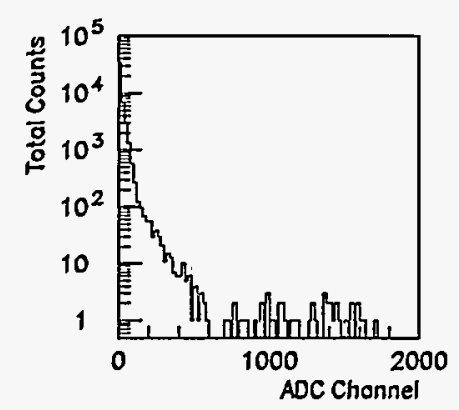

\#14

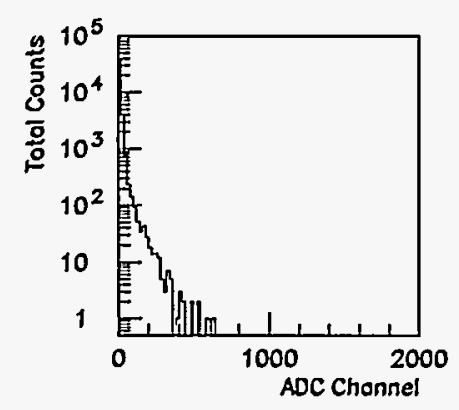

\#7
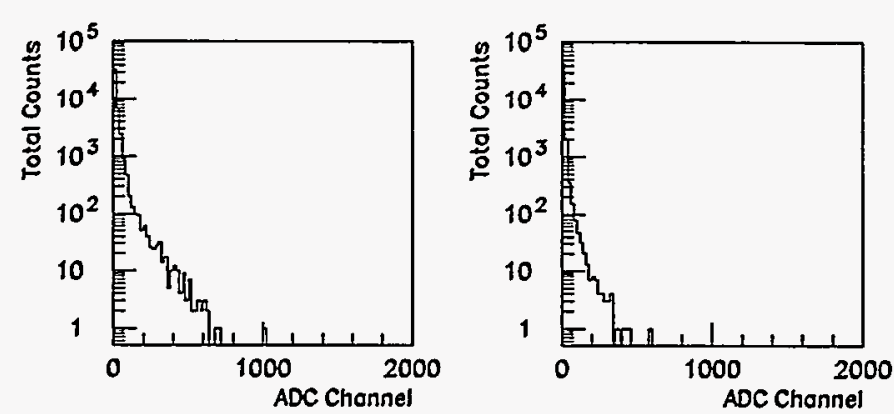

\#18

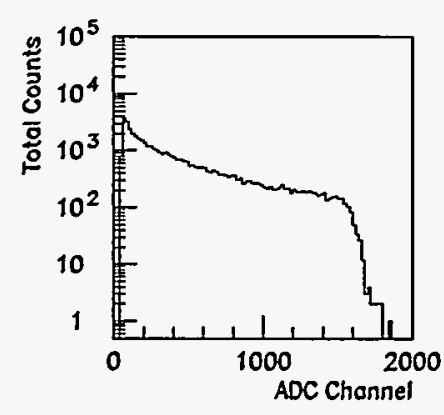

\#13

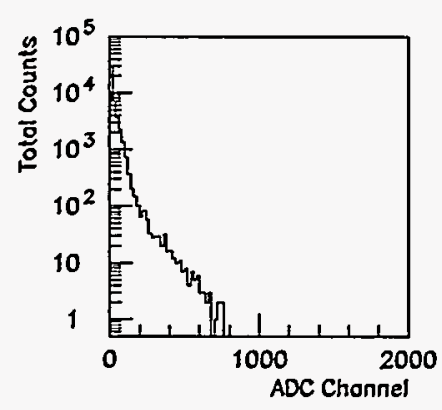

\#8

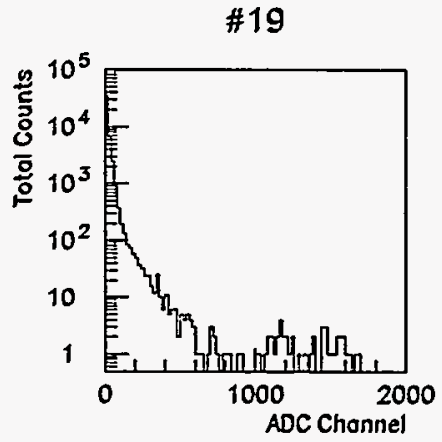

\#12

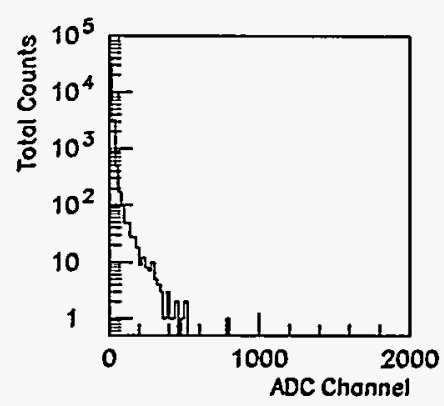

\#9

Figure 6: Typical uncorrected raw ADC pedestal-subtracted spectra from central $3 \times 3$ lead glass blocks of the calorimeter. The center block (\#13) contains $~ 92 \%$ of the energy deposited. Lateral energy spread is visible in all the surrounding blocks. The storage ring contained electrons at $60 \mathrm{~mA}$ during this data collection. 
energy resolution of the calorimeter. The Gaussian smearing is clear from the knee of the spectrum, which can be seen to be distributed over many ADC channels instead of sharply dropping at the cutoff limit. The functional form of the ADC distribution can be assumed as [16]:

$$
\frac{d F\left(N, C, \sigma_{0}\right)}{d(A D C)}=N \int_{E_{\gamma \min }}^{E_{\gamma \max }} \frac{1}{E^{3 / 2}} \exp \left\{\frac{-(E-A D C / C)^{2}}{2 \sigma_{0}^{2} E}\right\} d E
$$

where $E$ is the photon energy, $A D C$ is the measured $A D C$ value, $N$ is the normalization parameter, $\mathrm{C}$ is the energy scale factor (calibration constant), and $\sigma_{0}$ is the resolution of the calorimeter. The integral is evaluated from $\mathrm{E}_{\gamma \min }$, a minimum photon energy chosen, to $\mathrm{E}_{\text {rmax }}$, the maximum photon energy, which is the maximum particle energy. By fitting Equation 6 to the ADC bremsstrahlung spectrum, one obtains the values for $\mathrm{N}, \mathrm{C}$, and $\sigma_{0}$. To check this technique for the present scenario, a simple Monte Carlo simulation has been run to generate an ADC bremsstrahlung distribution with a calibration of 250 $\mathrm{ADC}$ channels $/ \mathrm{GeV}$ and a resolution of $10 \% / \sqrt{ } E_{\gamma}$ folded in. Based on observed values, a reasonable ADC pedestal and a lower energy threshold limit are also incorporated in the Monte Carlo. The ADC response thus obtained is shown in Figure 7a. Figure 7b shows a fit to the spectrum using Equation 6 with $\mathrm{E}_{\gamma \min }$ and $\mathrm{E}_{\gamma \max }$ values of 1 and $7 \mathrm{GeV}$, respectively. The fit results provided show that we can accurately unfold the calibration constant, as well as the resolution, of the calorimeter using the above relation.

Individual calibration constants of the lead glass blocks that comprises the $3 \times 3$ detector array were determined by fitting the corresponding ADC spectrum obtained from calibration data sets by Equation 6 . An arbitrary $\mathrm{E}_{\gamma \min }$ value of $2 \mathrm{GeV}$ was used. Using a single lead glass block and $7 \mathrm{GeV}$ photon beam, the (EGS4) [17] Monte Carlo simulation showed an average energy of only $6.1 \mathrm{GeV}$ being deposited in the medium due to incomplete shower containment. Therefore, $\mathrm{E}_{\text {ymax }}$ is chosen as $6.1 \mathrm{GeV}$ instead of the expected cutoff value of $7 \mathrm{GeV}$. The calibration constants $\mathrm{C}_{n}$ and the resolution values $\sigma_{n}$ obtained through the fits are given in Table 1 . The Calibration constant for the central detector 13 is approximately twice as much as the rest because of a factor of two less attenuation pre-set in this ADC channel to accommodate for half of its signal which has been split for the trigger circuit. The complete bremsstrahlung energy spectrum can 

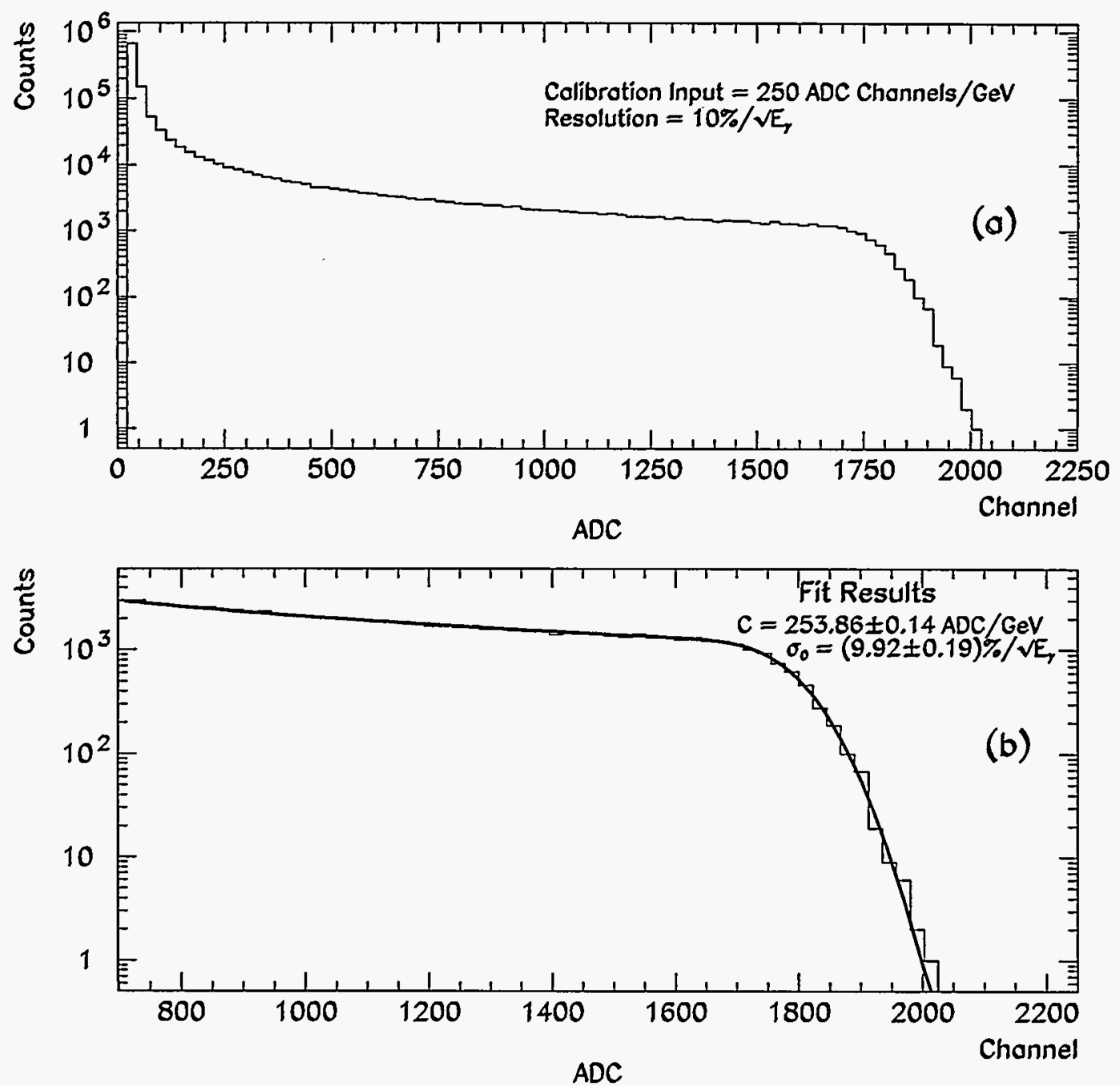

Figure 7: (a) An expected ADC distribution generated by monte carlo simulation with a calibration of $250 \mathrm{ADC} / \mathrm{GeV}$ and a resolution of $10 \% / \sqrt{ } E_{\gamma}$. (b) The endpoint of (a) with the fit described in equation (6). The fit results show that we can correctly measure the calibration constant $\mathrm{C}$ and the resolution $\sigma_{0}$ of the detector. 
Table 1: Lead glass detector calibration constants and resolutions as obtained from fitting corresponding ADC bremsstrahlung spectrum by Equation $6 . \mathrm{C}_{13}$ is approximately twice higher compared to the rest because of a factor of two less attenuation pre-set in this ADC channel to compensate for half of its signal used for the trigger.

\begin{tabular}{|c|c|c|}
\hline \hline $\begin{array}{c}\text { Lead Glass Detector } \\
\# \mathrm{n}\end{array}$ & $\begin{array}{c}\text { Calibration Constant } \\
C_{n} \\
(\mathrm{ADC} / \mathrm{GeV})\end{array}$ & $\begin{array}{c}\text { Resolution } \\
\sigma_{n} \\
\left(\% / \sqrt{ } E_{\gamma}\right)\end{array}$ \\
\hline$\# 7$ & $136.8 \pm 0.3$ & $6.8 \pm 0.6$ \\
\hline$\# 8$ & $140.2 \pm 0.3$ & $6.6 \pm 0.4$ \\
\hline$\# 9$ & $138.4 \pm 0.4$ & $7.6 \pm 0.7$ \\
\hline$\# 12$ & $138.7 \pm 0.3$ & $6.7 \pm 0.4$ \\
\hline$\# 13$ & $246.7 \pm 0.8$ & $8.0 \pm 0.7$ \\
\hline$\# 14$ & $115.0 \pm 0.2$ & $6.1 \pm 0.4$ \\
\hline$\# 17$ & $141.1 \pm 0.3$ & $6.1 \pm 0.5$ \\
\hline$\# 18$ & $138.9 \pm 0.6$ & $6.5 \pm 0.9$ \\
\hline$\# 19$ & $132.7 \pm 0.3$ & $8.5 \pm 0.6$ \\
\hline \hline
\end{tabular}

then be obtained using the relation:

$$
E=\sum_{n} \frac{1}{C_{n}}(A D C)_{n},
$$

where (ADC) $)_{n}$ are the raw spectra from the detectors as shown in Figure 6. Since the calibration constants given in Table 1 do not vary significantly from each other $(\leq 10 \%)$, it is possible to calculate a single effective calibration constant $\mathrm{C}_{\text {eff }}$ with reasonable accuracy, as $92 \%$ of the incident bremsstrahlung energy is contained in a single detector (the central detector). Thus:

$$
E=\frac{1}{C_{\text {eff }}} \sum_{n}(A D C)_{n}
$$

Because the electromagnetic showers from the events are $100 \%$ laterally contained within the $3 \times 3$ detector array, fitting the the summed spectrum of all the nine blocks would yield an effective calibration constant for the calorimeter. The only correction that 
needs to be applied in this method would be that due to longitudinal shower leakage out through the back side of the calorimeter.

In order to determine the effective calorimeter calibration constant, first ADC responses from each of the nine $3 \times 3$ detector array are added to obtain the total ADC spectrum. This distribution is then fit to Equation 6 with an $\mathrm{E}_{\text {ymin }}$ value of $2.0 \mathrm{GeV}$. $\mathrm{E}_{\gamma \max }$ is chosen as $6.77 \mathrm{GeV}$ instead of the expected $7 \mathrm{GeV}$ cutoff value, again based on results obtained from the electron gamma shower (EGS4) [17] Monte Carlo simulations. Using a $3 \times 3$ lead glass array and a $7 \mathrm{GeV}$ photon beam, an average energy of only $6.77 \mathrm{GeV}$ ( $96.8 \%$ of the total energy) was found to be deposited in the detector volume, the rest lost due to longitudinal shower leakage. As an example to demonstrate our calibration procedure and results, the total ADC spectrum from the experimental data acquired at a electron beam current of $60 \mathrm{~mA}$ is shown in Figure 8. The inset shows the cutoff region of the spectrum with the fit as discussed above. The fit unfolds effective calibration constant $C$ and resolution $\sigma_{0}$ of the calorimeter. These values are listed in the inset as fit results. The additional entries beyond the bremsstrahlung cutoff in the ADC total spectrum are most likely to be from real multiple bremsstrahlung events - events in which an electron bunch emits two photons simultaneously during their traverse through the $15.38 \mathrm{~m}$ straight path. Such events contribute to a distribution that is proportional to $1 / \mathrm{E}^{2}$, embedded within the normal $1 / \mathrm{E}$ bremsstrahlung spectrum. It is difficult to unfold such multiple photon-emitted events from the bremsstrahlung profile. Since the experiment cannot resolve these photons as originating from two separate events, only their added energy will be seen by the detector giving rise to entries having energies above the bremsstrahlung cutoff limit. That the additional events beyond the spectrum cutoff are indeed due to multiple photon emission process is discussed elsewhere in this chapter. Figure 9 shows the corresponding energy spectrum for the data in Figure 8. The ADC pedestals have been corrected for all the nine channels used in the analysis. The resulting bremsstrahlung distribution has the expected end point approximately at $6.77 \mathrm{GeV}$, smeared by the calorimeter resolution.

Similar fits conducted on data sets from various runs, yield a mean calibration constant $\left(\mathrm{C}_{e f f}\right)$ value of $250 \pm 10 \mathrm{ADC} / \mathrm{GeV}$ and a mean resolution value of $(10.0 \pm 0.7) \% / \sqrt{ } E_{\gamma}$. 


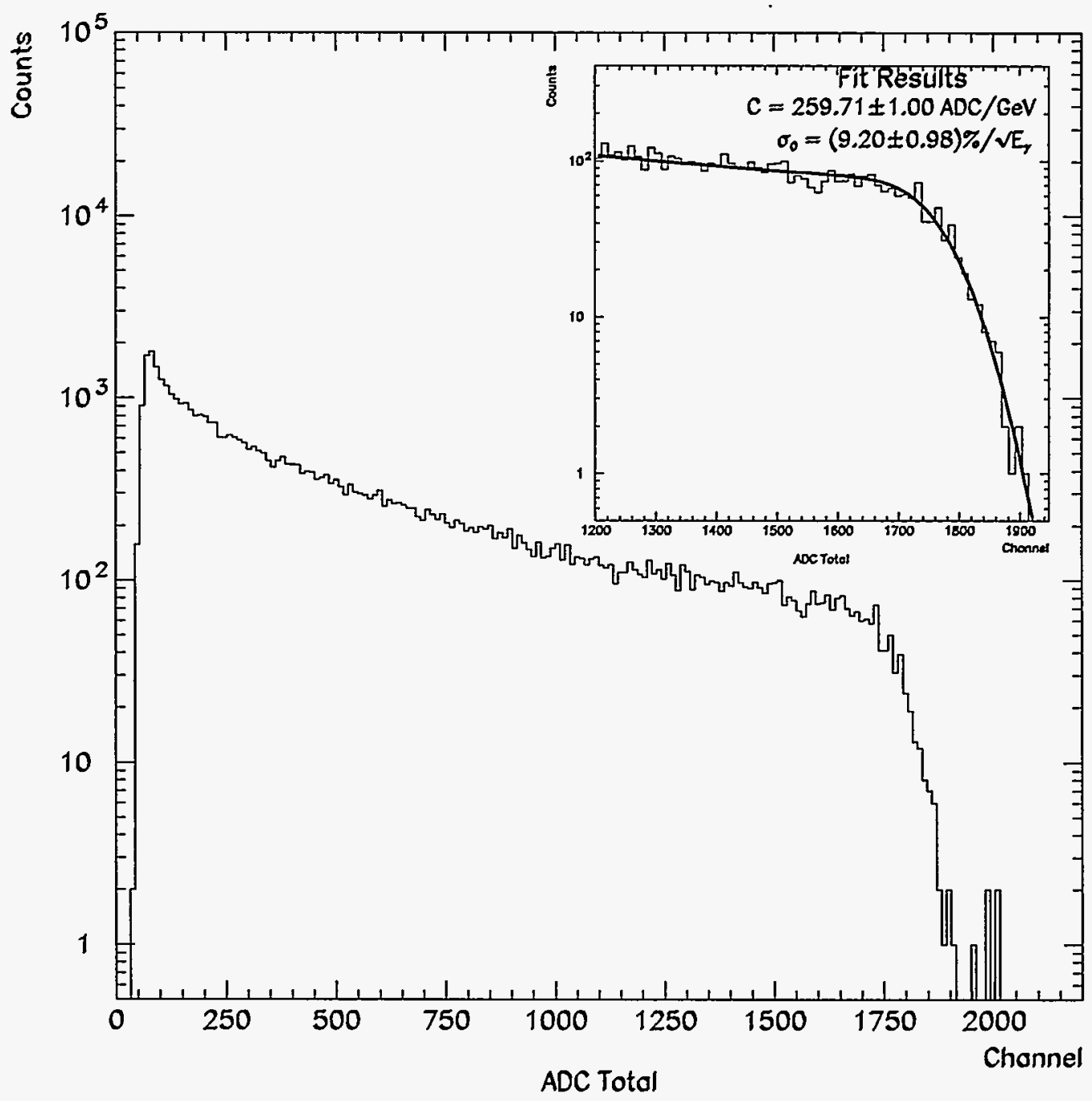

Figure 8: The summed ADC spectrum of all nine lead glass block detectors of the RE1 calorimeter. Additional entries after the cutoff are most likely to be from multi-bremsstrahlung events as discussed in the text. The inset shows the fit to the end-point region of the spectrum using Equation 6, which yields values for the calibration constant and resolution of the calorimeter. 


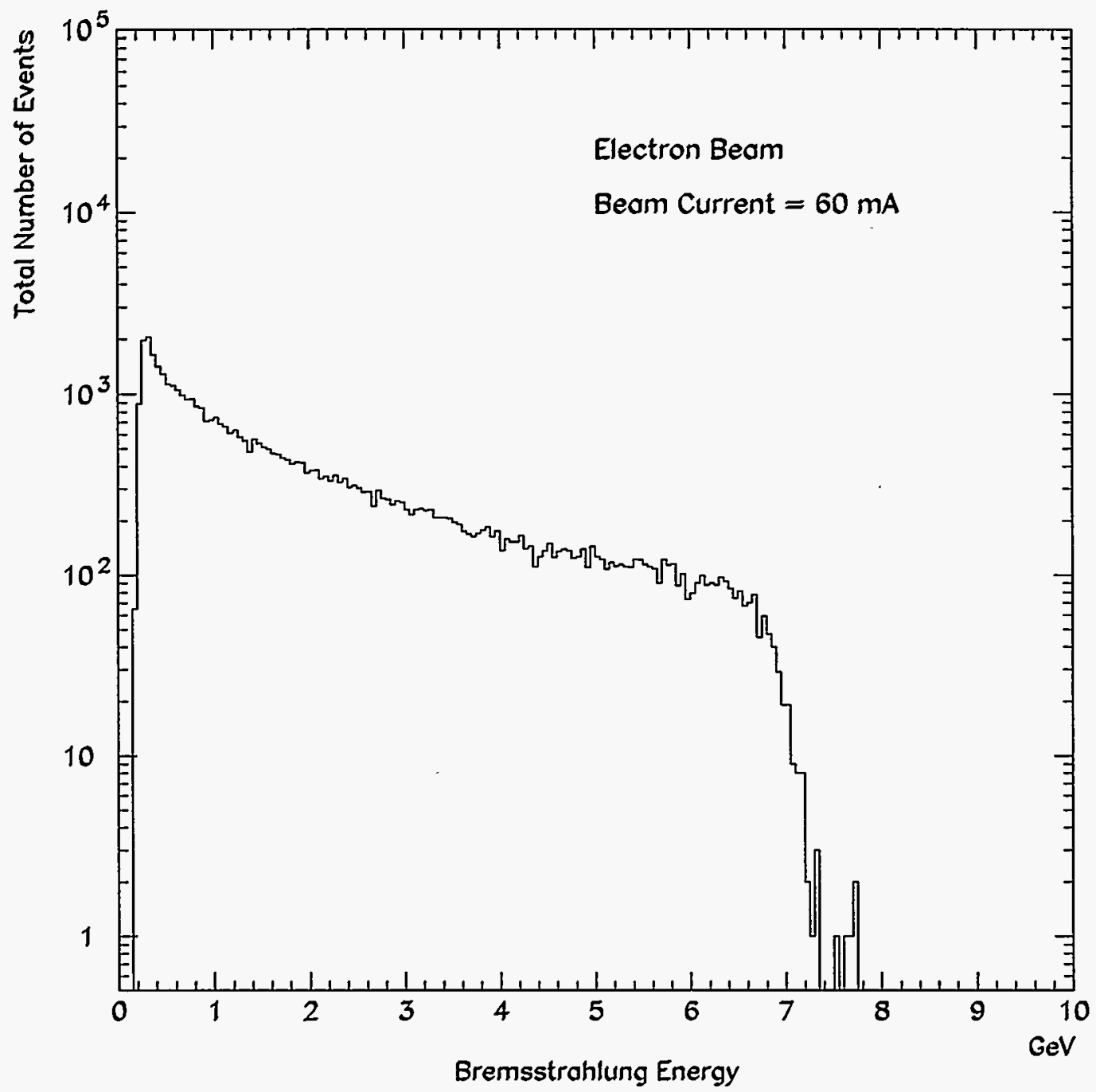

Figure 9: The uncorrected bremsstrahlung energy spectrum. Calibration constant $\mathrm{C}_{\text {eff }}$ obtained from the fit procedure has been used to convert the ADC distribution shown in Figure 7. The cutoff, smeared by the finite resolution of the calorimeter, can be seen at around $6.77 \mathrm{GeV}$. 


\subsection{Data Analysis}

In this section, a detailed description of the data analysis, both for electron as well as positron beam in the storage ring, are discussed. The nature of the bremsstrahlung spectrum, its production rate, and the bremsstrahlung energy radiated, all normalized to the effective vacuum pressure inside the $15.38 \mathrm{~m}$ storage ring straight path, are investigated for various beam currents. Because the gas bremsstrahlung is a function of the residual vacuum inside the straight section, accurate determination of the residual gas pressure is important for subsequent analysis. Several ion pump and ion gauge readings within the relevant storage ring straight paths are to be dealt with in order to obtain dependable vacuum values. The presence of vacuum fluctuations and instability also have to be investigated. We begin this section with matters related to vacuum values and their selection procedures before focusing our attention on the bremsstrahlung production rates and energy radiated. Various corrections that are to be applied to the results are then discussed and incorporated followed by investigations into the dose equivalent calculations from the radiated energy values.

\subsubsection{Vacuum Studies}

The intensity of gas bremsstrahlung is a function of the stored beam current and the residual gas pressure of the vacuum where it has been generated, as evident from the following equation $[3,10]$ :

$$
F_{k} d k=8.21 \times 10^{-11} Z(Z+1) p\left(\frac{273}{T}\right) I \frac{d k}{k} f\left(E, E_{0}, Z\right) \quad\left(\mathrm{cm}^{-1} \sec ^{-1}\right)
$$

where

$\mathrm{F}_{k} \mathrm{dk}=$ the number of photons of energy between $\mathrm{k}$ and $\mathrm{k}+\mathrm{dk}(\mathrm{MeV})$ emitted $/ \mathrm{sec} / \mathrm{cm}$, $\mathrm{Z}=$ atomic number of residual gas in the vacuum,

$\mathrm{p}=$ residual gas pressure of the vacuum (Torr),

$\mathrm{T}=$ temperature of the residual gas $(\mathrm{K})$,

$I=$ stored beam current $\left(\mathrm{e}^{-} / \mathrm{sec}\right.$ or $\left.\mathrm{e}^{+} / \mathrm{sec}\right)$,

$\mathrm{E}_{0}=$ total energy of the electron or positron $(\mathrm{MeV})$, and

$\mathrm{f}\left(\mathrm{E}, \mathrm{E}_{0}, \mathrm{Z}\right)=\left\{\left[1+\left(\frac{E}{E_{0}}\right)^{2}-\frac{2}{3} \frac{E}{E_{0}}\right] \ln \left(183 Z^{-\frac{1}{3}}\right)+\frac{1}{9} \frac{E}{E_{0}}\right\}$ 


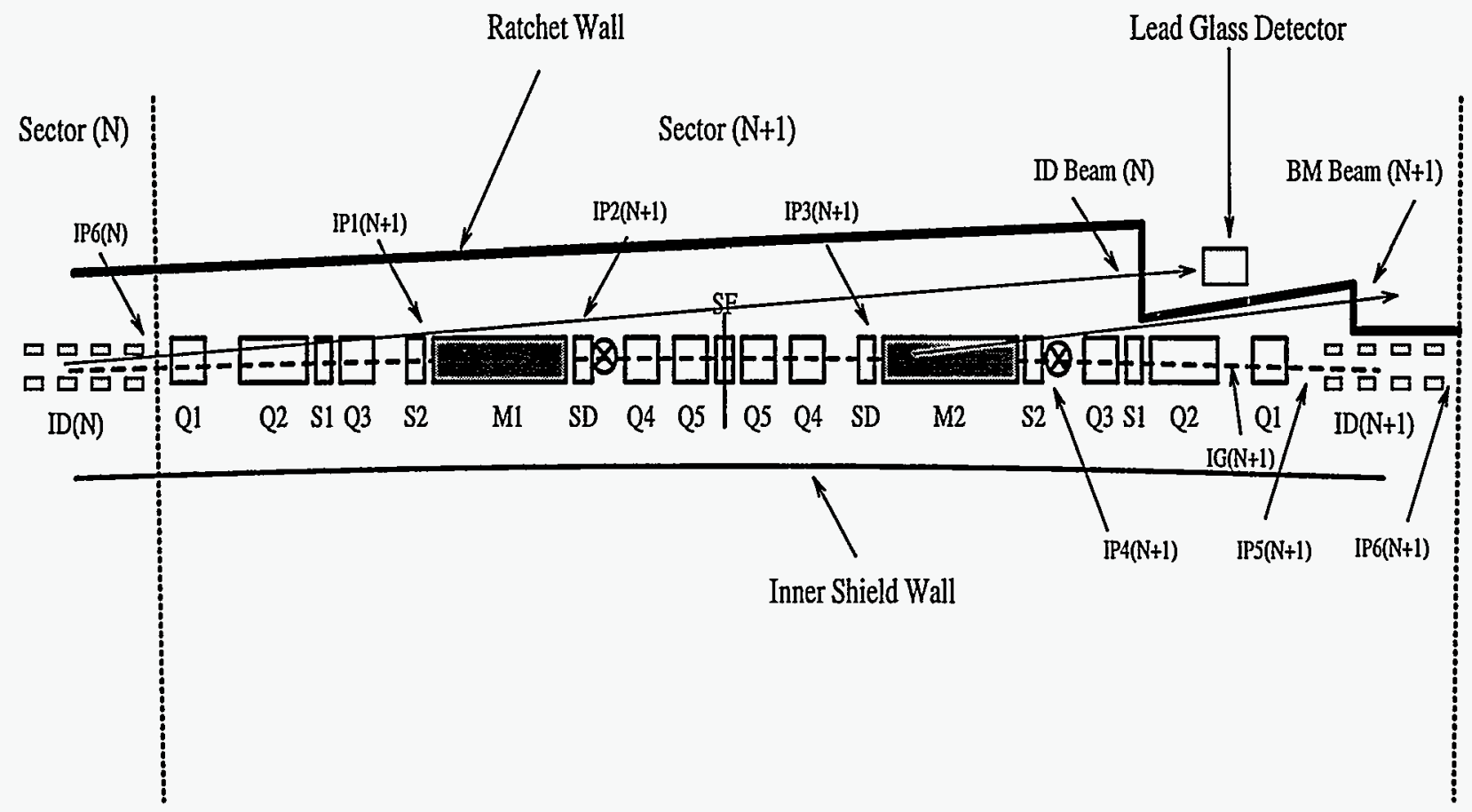

Figure 10: Layout of a sector $\mathrm{ID}(\mathrm{N})$ of the APS storage ring. The photon beamline from $\mathrm{ID}(\mathrm{N})$ traverses also through the sector $\mathrm{ID}(\mathrm{N}+1)$. Locations of various ion pumps (IP) and ion gauges (IG) that are in sector $N$ and $N+1$ are shown. The photon beamline of $I D(N)$ separates from the storage ring after the IP2 pump location of sector $(\mathrm{N}+1)$. The figure is not to scale.

Determination of the correct value of the vacuum is thus imperative for subsequent intensity and energy calculations. Bremsstrahlung photon(s) could be generated anywhere in a 15.38-m-long effective straight path that is in the line of sight of an ID beamline. These straight paths are equipped with ion pumps and gauges that provide information about the vacuum along the ID sector. The typical layout of one of the 40 sectors of the APS storage ring is shown in Figure 10. A sector consists of an insertion device (ID) and two bending magnets M1 and M2 along with several quadrupoles (Q) and sextupoles (S). The ID beamline of sector $N, \operatorname{ID}(N)$, goes through some length of the next sector $\mathrm{N}+1$. The total effective length of the straight path, which is the distance between $M 2(N)$ and $M 1(N+1)$, is $15.38 \mathrm{~m}$. Vacuum along this straight segment is monitored by six ion pumps (IP) and an ion gauge (IG), the locations of which are shown 
in the figure. Thus, the relevant information that is required to estimate a reasonably accurate vacuum for the $15.38 \mathrm{~m}$ storage ring straight path associated with an $\mathrm{ID}(\mathrm{N})$ should be extracted from readings of IP4(N), IG(N), IP5(N), IP6(N), IP1(N+1), and IP2 $(\mathrm{N}+1)$ at the data acquisition time.

The vacuum profiles of all the relevant IPs and IGs in the $15.38 \mathrm{~m}$ effective straight section of sector 12 (which includes part of sector 13 also) are shown in Figure 11 for a duration of 24 hours. The sudden drops in the profiles of IP4(12), IP5(12), IP2(13), and IG(12) correspond to zero beam current state (due to beam loss) of the storage ring. Maximum vacuum is attained under such zero beam current conditions. For non-zero beam currents, the average vacuum is typically of the order of a nTorr. The vacuum values, averaged over the data acquisition run time, are calculated from all these ion pump and gauge readings. The straight path segment-length weighted values of these time-averaged individual vacuum readings are then used for normalizing various results. Although vacuum fluctuations are present on a longer time scale, as shown in Figure 11, no significant variations $(>10 \%)$ are observed over the typical data collection time period of 16 minutes. The vacuum readings in nTorr from two ion pumps and an ion gauge of Figure 11 are shown in Figure 12 for a typical data collection duration. In the final analysis, systematic errors in the vacuum readings were not taken into consideration.

\subsubsection{Multiple Bremsstrahlung Events}

As briefly mentioned in section 4.2 , for a bunch of electrons, a non-zero probability exists of emitting multiple photons simultaneously during their traverse through the $15.38 \mathrm{~m}$ straight path. The probability for this to occur at the storage ring vacuum has been estimated to be less than $1 \%$ by Monte Carlo studies [18].

The double bremsstrahlung production will roughly follow a $\frac{1}{E^{2}}$ distribution. These events are embedded within the $\frac{1}{E}$ bremsstrahlung profile and are difficult to distinguish, except beyond the $7 \mathrm{GeV}$ cutoff. Because the experiment cannot resolve these simultaneously generated photons, only their added total energy is detected by the calorimeter and the data acquisition system. This results in the presence of what appears to be extra pileup events after the cutoff of the $\frac{1}{E}$ bremsstrahlung spectrum. This effect is observed in the spectrum given in Figure 9. It shows the gas bremsstrahlung spectrum, 

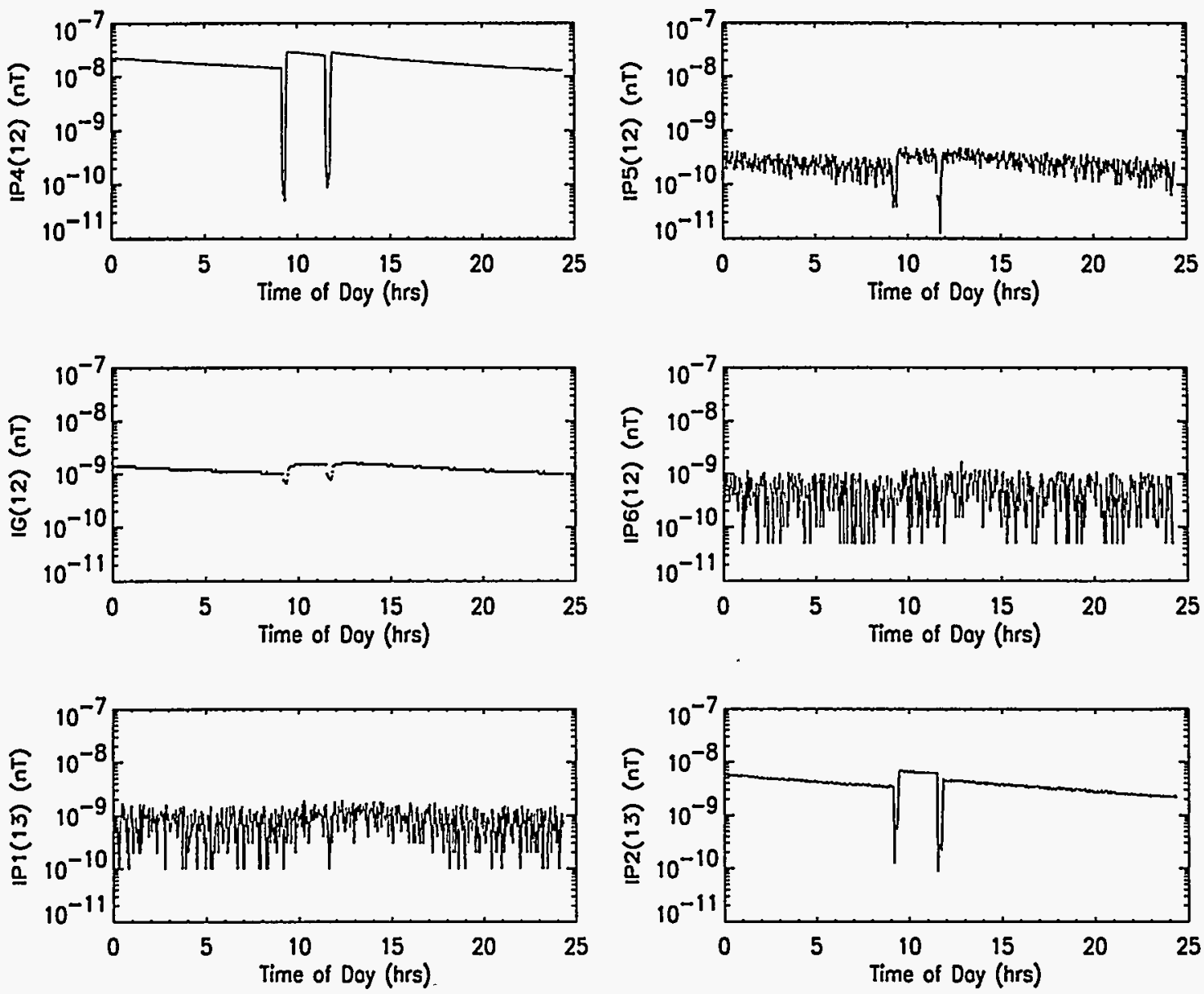

Figure 11: The typical vacuum value profiles (in nTorr) from sector 12 (IP4(12), IP5(12), IG(12), and IP6(12) ) and sector 13 (IP1(13) and IP2(13)), shown for a period of 24 hrs. The storage ring had been operating with electron beam during the hours of the day shown here. 


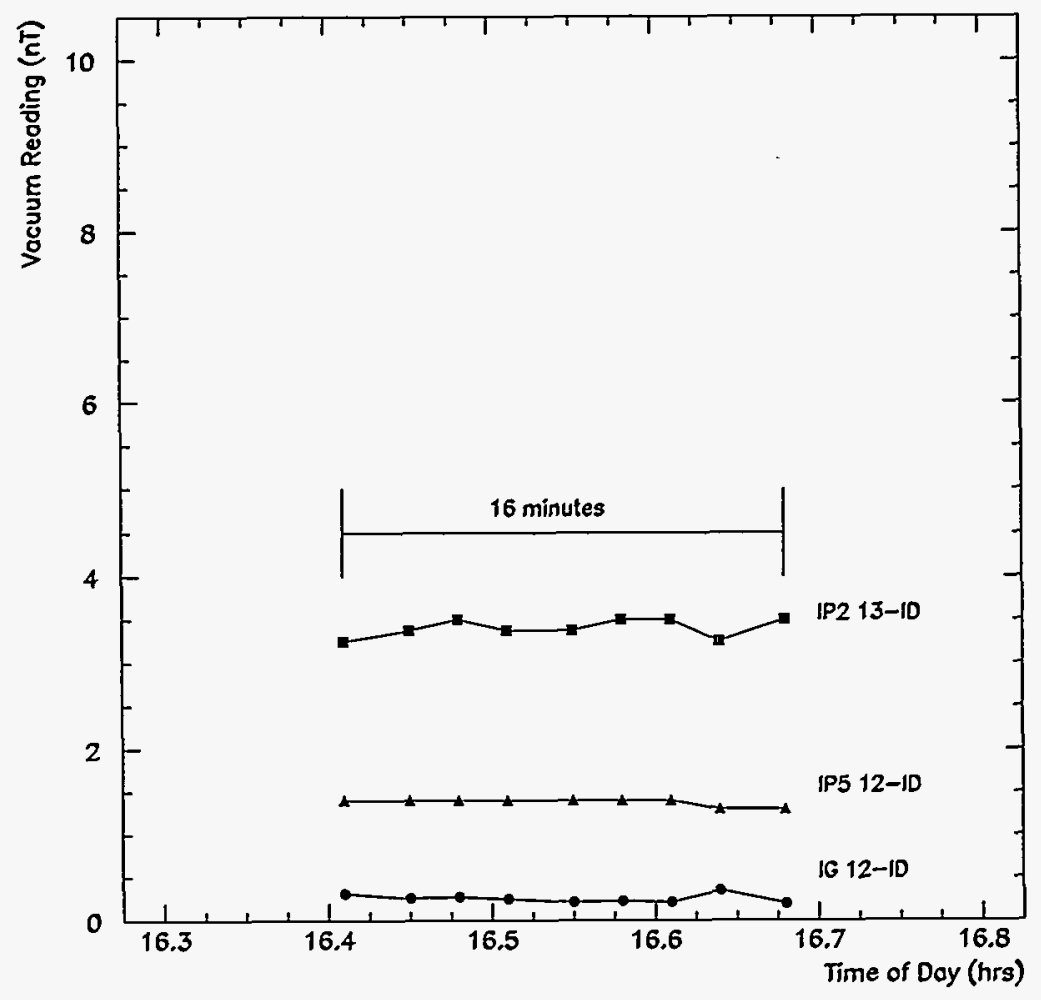

Figure 12: The vacuum readings (in nTorr) from IP2(13), IP5(12), and IG(12) for a typical data collection duration time of 16 minutes. No significant variations are observed in the vacuum values during the data collection interval. 
calibrated to the energy scale, for a $60 \mathrm{~mA}$ electron beam in the storage ring. The additional entries beyond the resolution-smeared knee at around $6.77 \mathrm{GeV}$ can be attributed to multiple photon production. These events actually represents the tail end of the $\frac{1}{E^{N}}$ distribution, where $\mathrm{N}=2$ is most likely. A Monte Carlo simulation has been run to see the result of a $\frac{1}{E}$ distribution superimposed with a $\frac{1}{E^{2}}$ profile. Realistic values equivalent to observed ADC pedestals and detector resolution are put into the simulation. One hundred thousand entries are generated, of which $1 \%$ are selected to be double emission events. The resulting spectrum is shown in Figure 13. The spectrum indicated by the solid line represents the pure $\frac{1}{E}$ distribution with a $10 \%$ resolution folded in. In this pure $\frac{1}{E}$ distribution, $1 \%$ of all the events is then selected for double emission. The $\frac{1}{E^{2}}$ profile thus obtained is then superimposed with the $\frac{1}{E}$ spectrum resulting in the distribution shown with the dotted lines. Extra events, which constitute the tail of the $\frac{1}{E^{2}}$ spectrum, are now visible beyond the cutoff. The effect does not seem to shift the knee of the spectrum. Thus the end point of the spectrum can still be fit as accurately as described in the calibration procedure section, even in the presence of such extra events near the cutoff region.

\subsubsection{The Residual Gas Analysis}

A detailed analysis of the nature of the residual gas (RG) inside the storage ring has been conducted at different sectors, and a summary of the results are presented in Table 2. Several RG measurements are conducted for two different situations - with the beam $\mathrm{ON}$ in the storage ring and with no beam in the storage ring. The results shown in Table 2 represent an average of all such measurements that are performed. In both the beam on and off states, $\mathrm{CO}$ appears to be the major component by weight constituting the residual gas inside the vacuum chamber, followed by almost identical levels of $\mathrm{CO}_{2}$ and $\mathrm{H}_{2}$. From the results, an effective $\mathrm{Z}$ for the residual gas inside the vacuum chamber is calculated approximately as 4.6 when there is beam in the storage ring. For atmospheric air, the effective $\mathrm{Z}$ value is approximately 7.2. A leak in the storage ring vacuum therefore could cause an enhancement in the bremsstrahlung production, which, in turn, effectively introduces a non-linear effect on the production rate with respect to the vacuum. 


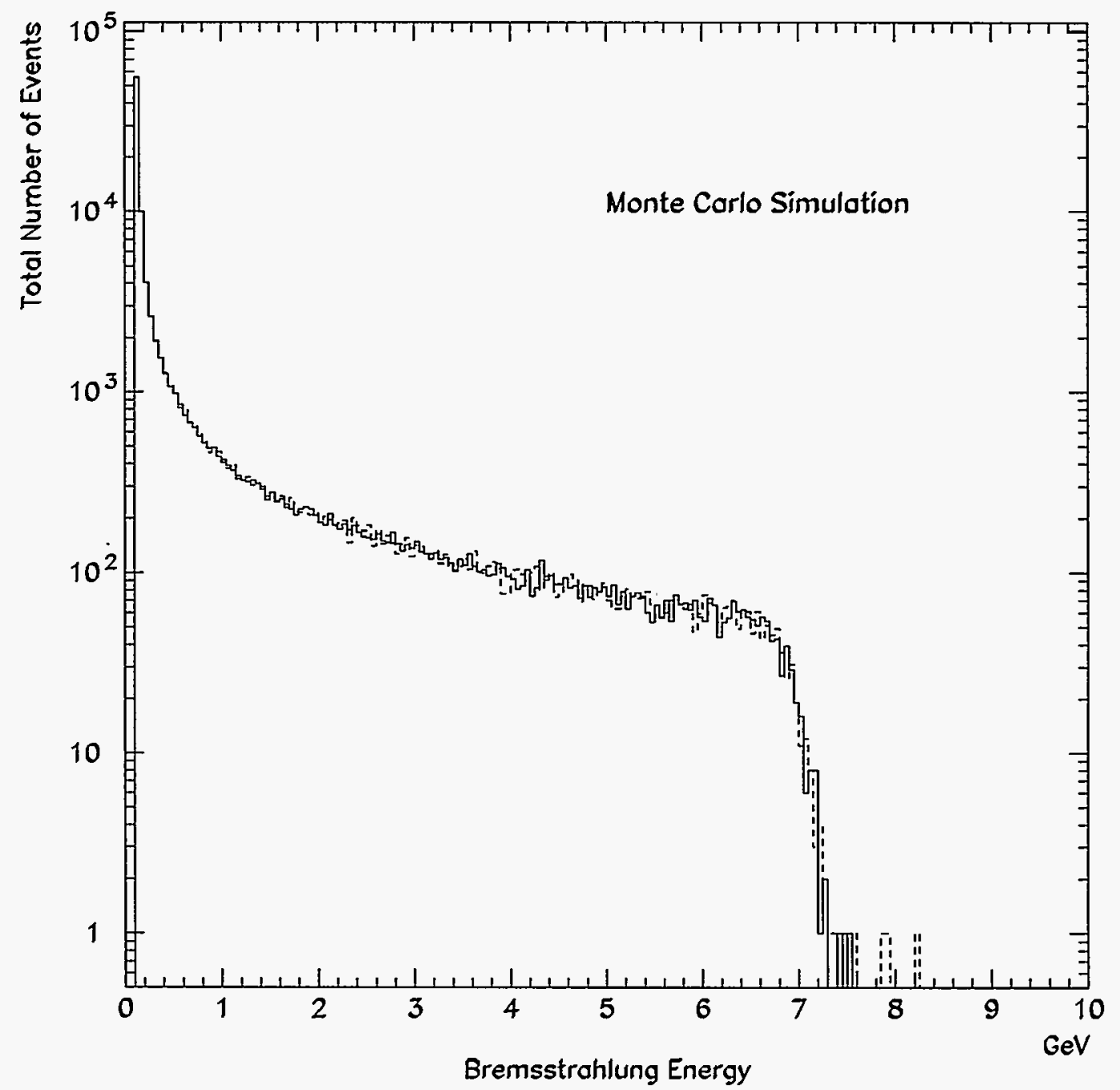

Figure 13: Simulated bremsstrahlung spectra. The solid line is the $\frac{1}{E}$ spectrum with detector resolution smearing. The spectrum represented by the dotted line is the superposition of $\frac{1}{E}$ as well as a $1 \% \frac{1}{E^{2}}$ entries of the total number of events. The extra events in the dotted spectrum beyond the cutoff are visible. 
Table 2: Summary of the RGA studies.

\begin{tabular}{|c|c|c|c|c|c|}
\hline \hline \multirow{2}{*}{$\begin{array}{c}\text { Mass } \\
\text { Number }\end{array}$} & $\begin{array}{c}\text { Residual Gas } \\
\text { Component }\end{array}$ & \multicolumn{2}{|c|}{$\begin{array}{c}\text { Mole Fraction } \\
(\%)\end{array}$} & \multicolumn{2}{c|}{$\begin{array}{c}\text { Weight Percent } \\
(\%)\end{array}$} \\
\cline { 3 - 6 } & & Beam on & Beam off & Beam on & Beam off \\
\hline 2 & $\mathrm{H}_{2}$ & 70.1 & 73.0 & 15.0 & 17.6 \\
\hline 12 & $\mathrm{C}$ & 0.88 & 0.48 & 1.10 & 0.00 \\
\hline 16 & $\mathrm{CH}_{4}$ & 1.70 & 2.30 & 2.90 & 4.40 \\
\hline 17 & $\mathrm{OH}$ & 0.75 & 0.97 & 1.40 & 2.00 \\
\hline 18 & $\mathrm{H}_{2} \mathrm{O}$ & 2.13 & 3.43 & 4.10 & 7.40 \\
\hline 28 & $\mathrm{~N}_{2} / \mathrm{CO}$ & 19.0 & 15.7 & 57.1 & 53.1 \\
\hline 40 & $\mathrm{Ar}$ & 0.30 & 0.31 & 1.30 & 1.50 \\
\hline 44 & $\mathrm{CO}_{2}$ & 3.60 & 2.60 & 17.1 & 14.0 \\
\hline \hline \multicolumn{5}{|c|}{}
\end{tabular}

\subsubsection{The Measured Bremsstrahlung Energy Spectrum}

A total of four different measurements of the gas bremsstrahlung intensity were conducted at various beamlines during a time interval of five months. Two were performed at ID sector 12 with electron beam in the storage ring. The ID sector 10 was used for the third measurement, and the ID sector 13 for the fourth one, both with stored positron beam. The data from all the four runs were analyzed, and the results are presented separately in this section.

The photon production rate is determined by using the scalers that were written out in the data. Since scaler $2\left(\mathrm{~S}_{2}\right)$ gives the raw events written out in the time governed by the pulser (scaler $S_{1}$ ), which is set at $10 \mathrm{KHz}$, the raw rate of events $\mathrm{N}^{i}$ in the $\mathrm{i}^{\text {th }}$ memory buffer (photons/sec/buffer) is obtained by weighting the corresponding energy spectrum of the $\mathrm{i}^{\text {th }}$ buffer by the factor $\mathrm{W}^{i}$ which is given by:

$$
W^{i}=\frac{1}{n} \frac{S_{2}^{i}}{S_{1}^{i} \times 10^{4}}
$$

where $n$ is the total number of events in the $i^{\text {th }}$ buffer. The sum of these weighted individual buffer rate spectra, averaged over the total number of buffers $b$ used for the analysis, yields the spectrum for the average photon production rate $\langle\mathrm{N}\rangle$.

$$
<N>=\frac{\sum_{i=1}^{i=b} N^{i}}{b}
$$


Figure 14 (a) through (d) shows the gas bremsstrahlung production rate in energy scale, for a few selected beam currents, from all the four different experimental runs mentioned earlier. The $y$-axis gives the average production rate $\langle N\rangle$ determined by Equations (10) and (11), normalized to the estimated vacuum, of photons having corresponding energies $\mathrm{E}_{\gamma}$ shown on the $\mathrm{x}$-axis. The results shown in Figure 14, are corrected for the data acquisition system dead time ${ }^{1}$ and $A D C$ pedestals. Some multiple photon production is visible in various spectra from all four runs. The total number of bremsstrahlung photons emitted in units of $(\mathrm{sec})^{-1}(\mathrm{nT})^{-1}$ are now determined for the corresponding beam currents using the relation:

$$
N_{\gamma}^{t o t}=\sum_{E_{\min }}^{E_{\max }} \Delta N
$$

where $\Delta N$ is the photon production rate at a given energy. The bremsstrahlung energy radiated in units of $(\mathrm{GeV})(\mathrm{sec})^{-1}(\mathrm{nT})^{-1}$ are also calculated for corresponding beam currents by multiplying photon energies on the $\mathrm{x}$-axis with the corresponding rate on the $y$-axis and summing over the entire energy range.

$$
E_{\gamma}^{t o t}=\sum_{E_{\min }}^{E_{\max }} E_{\gamma} \Delta N
$$

The finite sum values calculated from Equation (12) and Equation (13) include both single and multiple photon events, as well as their energies. The detailed results obtained along with the respective vacuum values are tabulated in Table 3 . The low energy threshold and the longitudinal shower leakage corrections, which will be discussed in the following section, are yet to be applied to the numbers representing the total energy radiated in Table 3.

\subsection{Systematic Errors}

Corrections to the energy spectra in Figure 14, for the systematic errors, are discussed in the following few sections. First the already applied dead-time correction procedure is outlined, followed by estimation and subsequent application of the longitudinal shower leakage and low energy threshold corrections.

\footnotetext{
${ }^{1}$ see section 4.4 .1 for a detailed description
} 

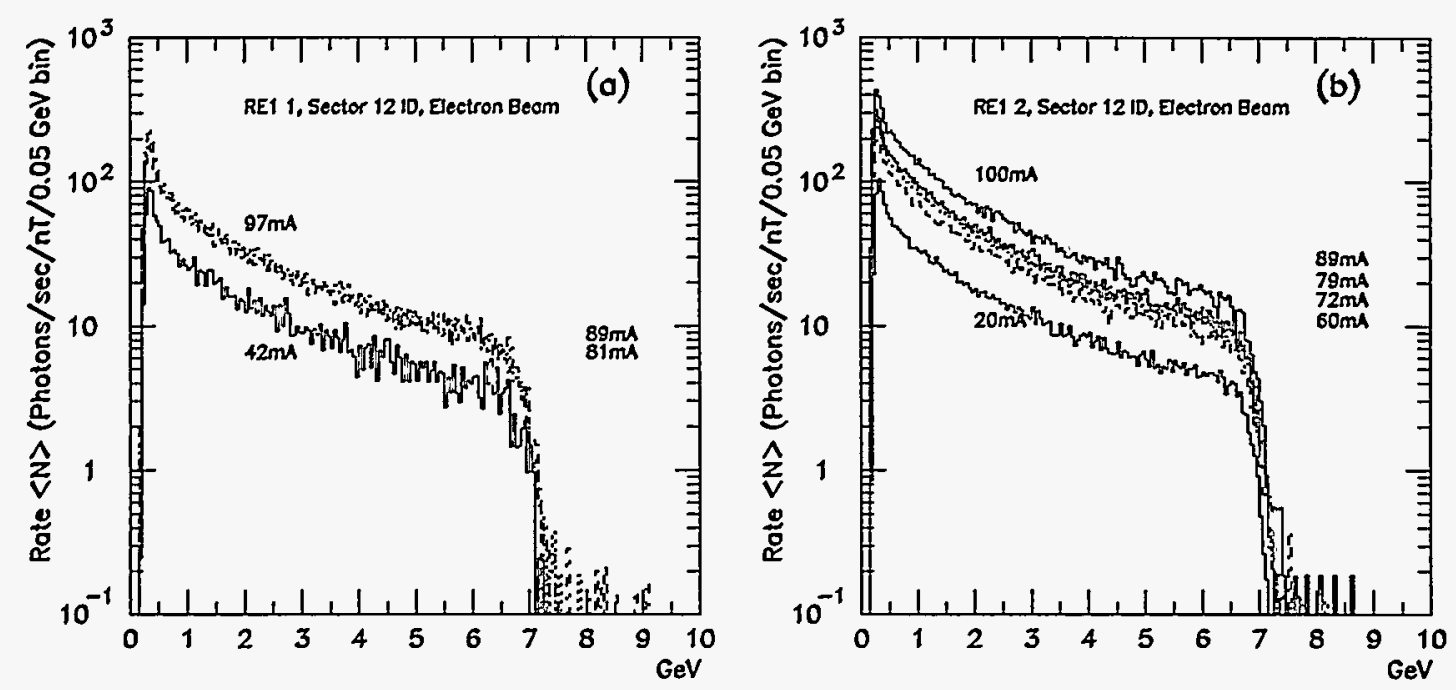

Photon Energy $\left(E_{\text {p }}\right)$

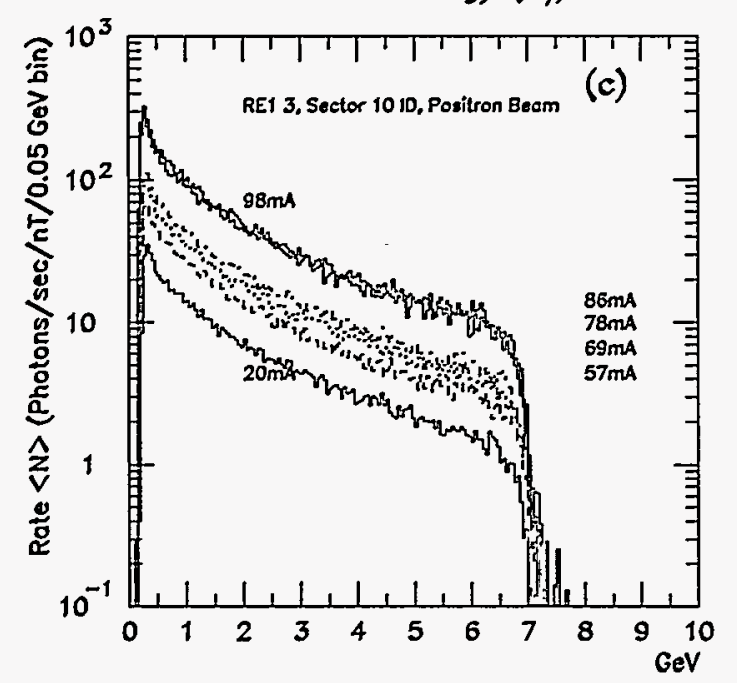

Photon Energy (Ey)

Photon Energy $\left(E_{\gamma}\right)$

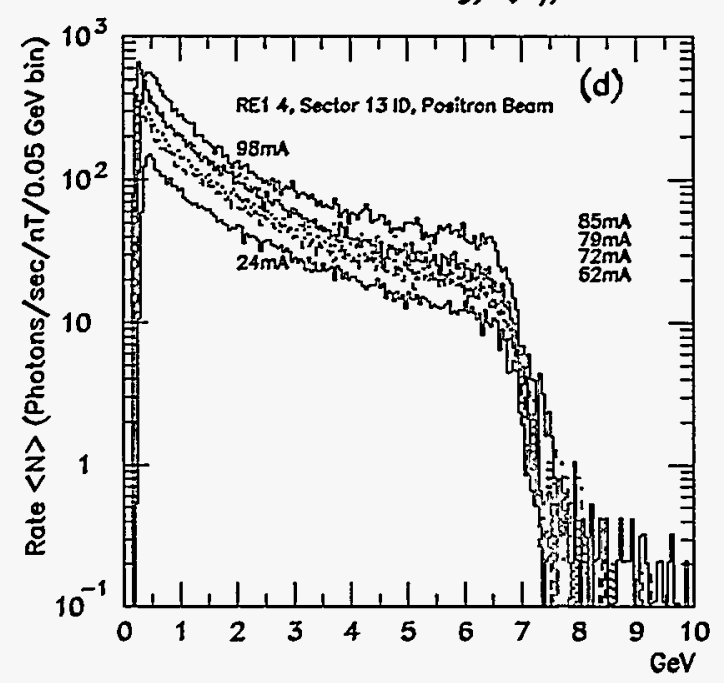

Photon Energy $\left(E_{y}\right)$

Figure 14: The gas bremsstrahlung energy spectra, corrected for dead-time losses, for typical beam currents from the electron beam runs \#1 and \#2 in sector 12 ID (Figures $\mathrm{a}$ and $\mathrm{b}$ ) and from the positron beam runs \#3 in sector 10 ID and \#4 in sector 13 ID (Figures $c$ and $d$ ). The $y$-axis represents the photon production rate $\langle N\rangle$ normalized to the vacuum. The lowest and highest beam currents are marked by the respective spectra, and the currents for the intermediate plots are given on the side in that order. 
Table 3: Observed dead-time-corrected bremsstrahlung energy originating from the 15.38 meter particle beam straight path (not corrected for other systematic errors).

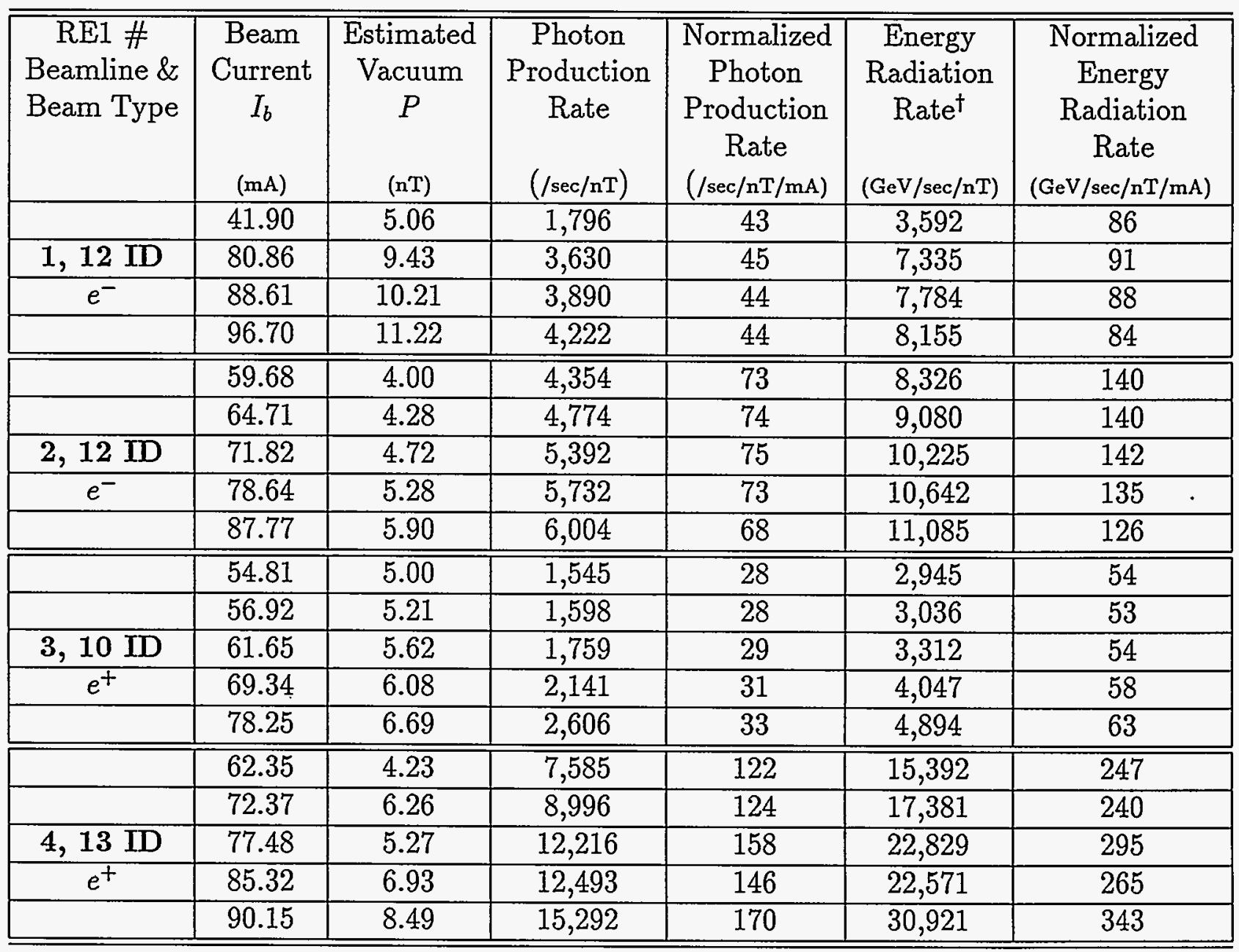

$\dagger \pm 0.4 \%$ statistical error. 


\subsubsection{Dead-Time Correction}

As mentioned earlier, the energy spectra in Figure 14 are already corrected for deadtime effects of the data acquisition system. Equation 5 is used to calculate the required correction. The value for $\tau$ in the equation is taken as $15 \mu \mathrm{sec}$, which has been pre-set in the data acquisition system through a gate and delay module.

To demonstrate the effect of dead-time correction, energy spectra for two typical beam currents are chosen and Equation 5 is applied. The resulting corrected spectra, superimposed on the corresponding uncorrected ones, are shown in Figure 15. The figure is plotted in linear scale along the y-axis in order to be able to clearly distinguish between the unhatched (corrected) and hatched (uncorrected) distributions. Evidently, as can be seen from Equation 5, the correction is relevant only at high rates for the $\tau$ value that has been selected. This can be seen at the low energy ends of the spectra in both Figure $15 \mathrm{a}$ and $\mathrm{b}$, where the rate of photon production is high. The effect of the dead-time correction becomes totally insignificant as we go to the high energy end of the spectrum. Between Figure 15a and b, the dead-time effect is greater in (b) than in (a), which is at a lower beam current compared to (b). On an average, a maximum of $3.0 \%$ correction is observed for typical data corresponding to the largest beam current. The dead-time correction is found to be insignificant for data from low currents.

\subsubsection{Longitudinal Shower Leakage Correction}

In order to completely contain an electromagnetic shower in the longitudinal direction, a minimum of $20 \mathrm{X}_{0}$ material of the shower medium is required. Shower leakage through the back side of our $13.5 \mathrm{X}_{0}$ calorimeter is therefore possible, requiring necessary correction to the estimated total bremsstrahlung energy radiated values in Table 3.

An EGS4 [17] Monte Carlo simulation has been run to understand the correlation between the incoming bremsstrahlung energy and the fraction of it leaking out through the back of the lead glass calorimeter. The geometry selected consists of lead glass blocks, each $35 \mathrm{~cm}$ long, in a $3 \times 3$ array. A total of 1000 bremsstrahlung events are then generated and allowed to be traced through the simulated geometry. Figure 16 shows the incoming energy (a), the leakage energy spectrum at the back side (b), and 

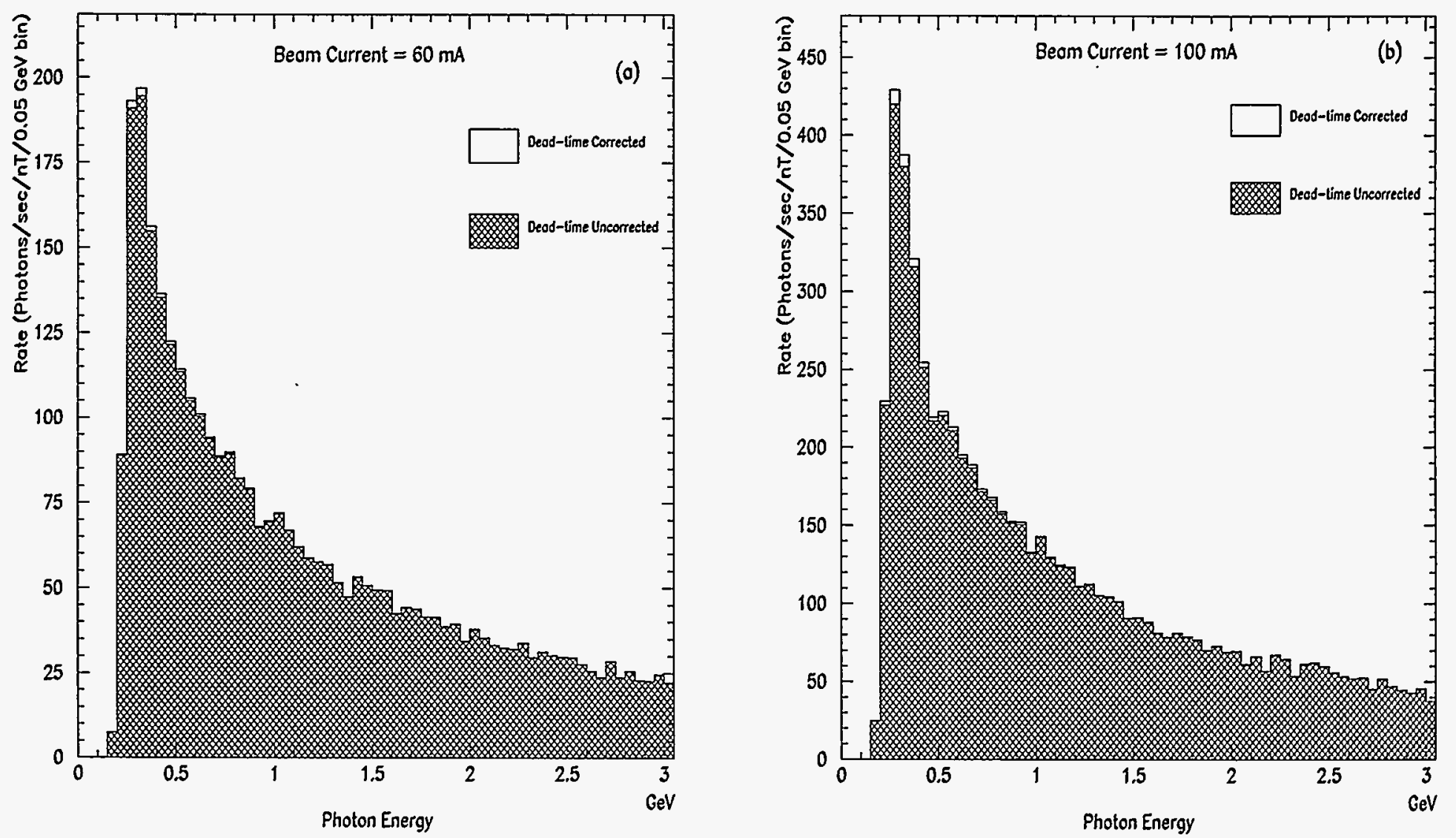

Figure 15: Energy spectra uncorrected and corrected for dead-time from the electron run \#2 in sector $12 \mathrm{ID}$, for $60 \mathrm{~mA}$ (a) and $100 \mathrm{~mA}$ (b) beam currents. 

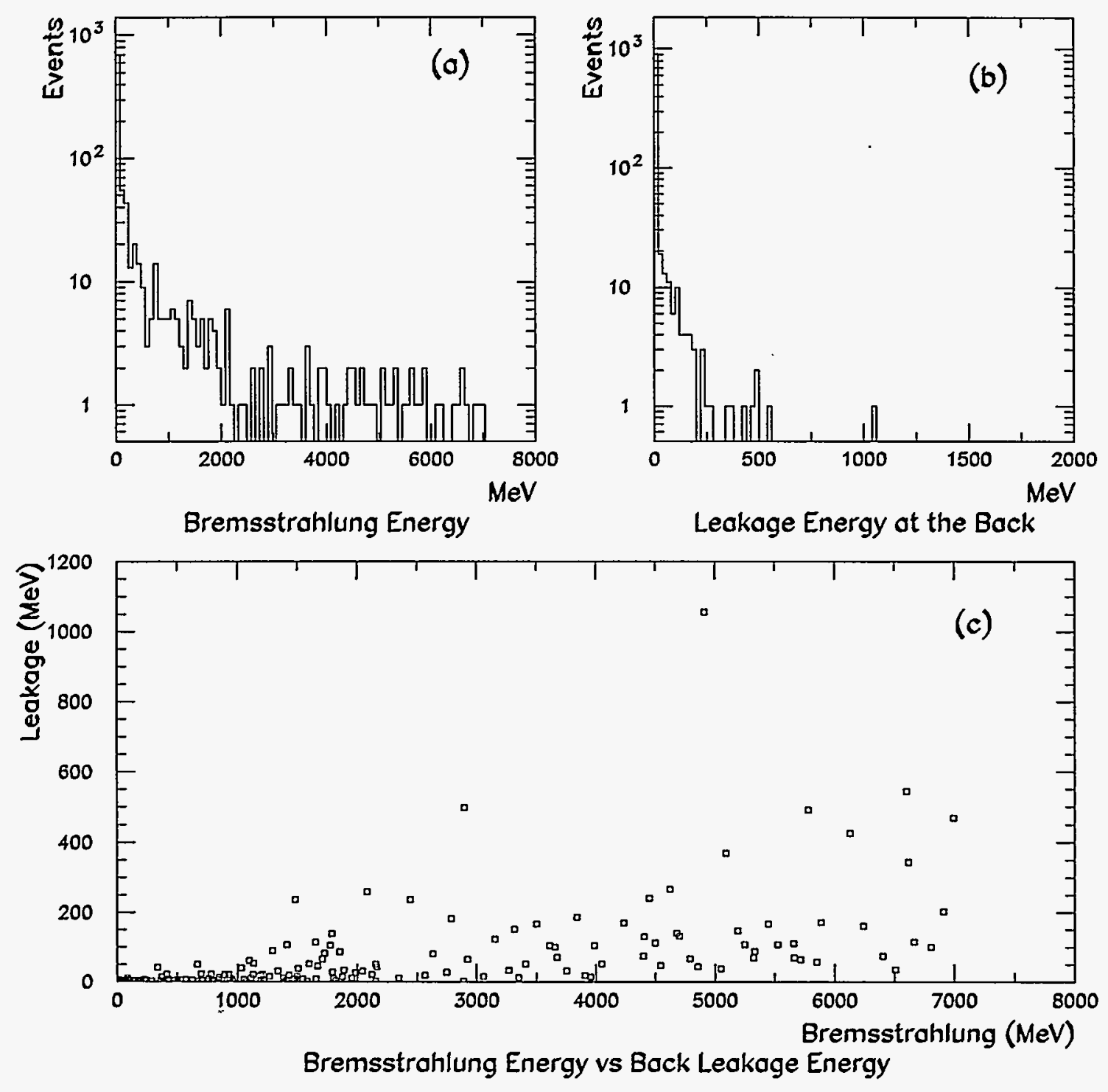

Figure 16: EGS4 Monte Carlo results. (a) Incoming bremsstrahlung energy spectrum. (b) The shower leakage energy profile at the back side of the RE1 calorimeter. (c) Scatter plot between (a) and (b) showing the correlation. 
a scatter plot between (a) and (b) showing their correlation. From Figure 16(c), it is clear that leakage at the back side is zero at low incident bremsstrahlung energies (below $1 \mathrm{GeV}$ ). Thus electromagnetic showers generated by incoming photons below 1 $\mathrm{GeV}$ are fully contained by the calorimeter. Above $1 \mathrm{GeV}$ however, Figure 16(c) shows that leakage begins to occur through the rear side of the calorimeter. On an average, approximately $4.3 \pm 0.1 \%$ of the incoming energy is estimated to be leaking out through the back end of the calorimeter at energies above $1 \mathrm{GeV}$. This average percent fraction is used to account for the longitudinal shower leakage. The average leakage energy in the lateral direction is found to be zero.

\subsubsection{Low Energy or Threshold Correction}

In order to eliminate residual synchrotron background, aside from the copper shield placed in front of the calorimeter, a discriminator threshold has also been pre-set as discussed in section 2.3. This threshold setting cuts off events having energy approximately below $150 \mathrm{MeV}$. Along with the synchrotron events, some low energy bremsstrahlung will also be lost in this setup. The lost bremsstrahlung energy has to be corrected for in the spectrum analysis results shown in Table 3.

To estimate the missing bremsstrahlung energy below the threshold setup, a fit to the spectrum of interest in Figure 14 is performed. It is convenient to convert the $\left\langle N>\propto\left(\frac{1}{E_{\gamma}}\right)\right.$ distributions in Figure 14 to a constant flat spectrum using the following relation:

$$
E_{\gamma} \times<N>\propto E_{\gamma} \times \frac{1}{E_{\gamma}}=\text { Constant }
$$

Equation (14) will yield a more or less flat distribution, which can then be easily fit. Examples of such converted spectra from Figure 14(a) through (d), for a single beam current in each, are shown in Figure 17.

A straight line fit to one of the typical distributions in Figure 17, along with the resulting fit parameters, is shown in Figure 18. Integrating the fit function along the entire energy range of the spectrum (entire number of bins of the histogram, since the $\mathrm{y}$-axis is per $0.05 \mathrm{GeV}$ bin) will then give us the total energy radiated in $\mathrm{GeV}$ per sec per nT. Conversely, the same fit function can be integrated in the energy region $0 \mathrm{GeV}$ to $0.2 \mathrm{GeV}$ or over the initial four bins (threshold cutoff region) in a similar manner to 

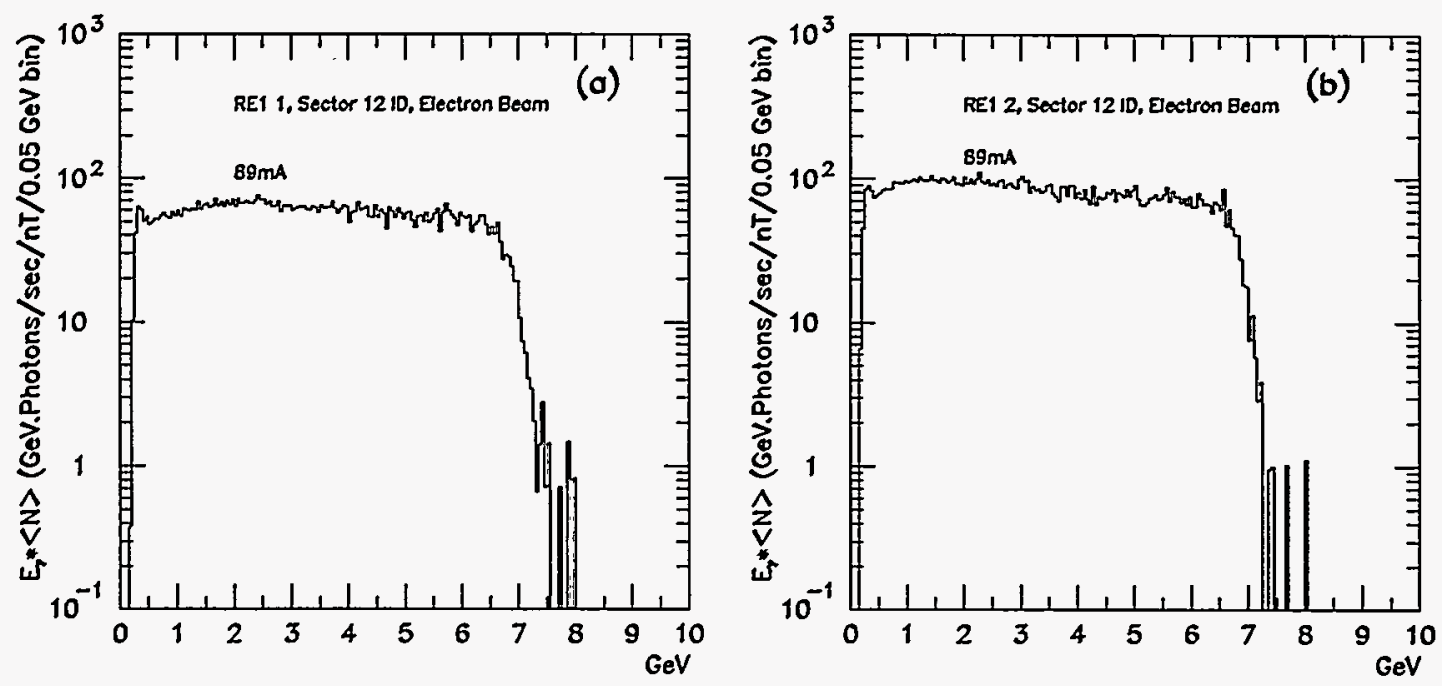

Photon Energy ( $E_{\text {, }}$ )

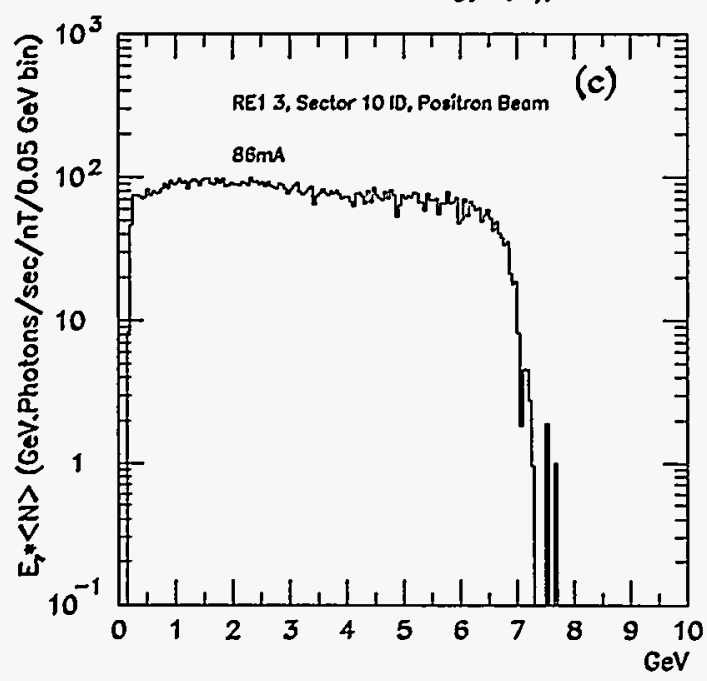

Photon Energy $\left(E_{r}\right)$

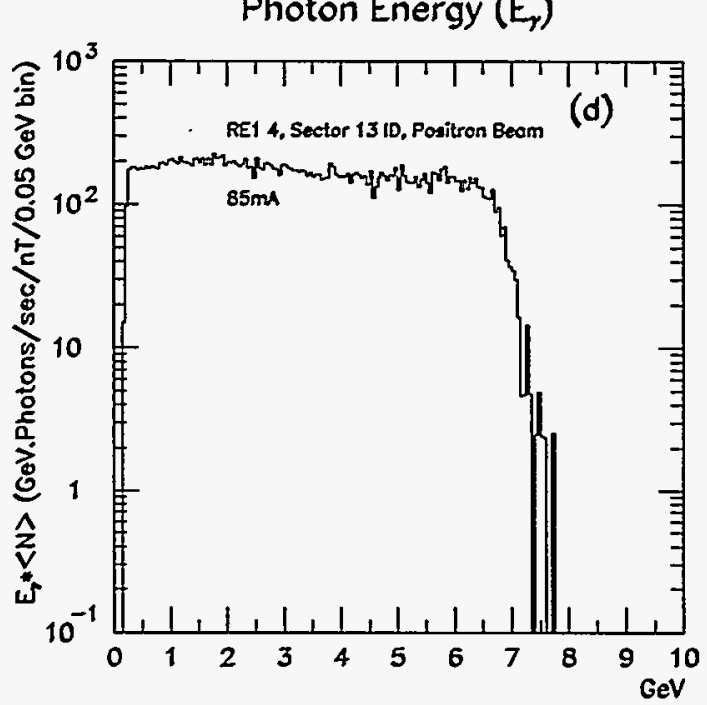

Photon Energy $\left(E_{y}\right)$

Figure 17: Typical dead-time corrected bremsstrahlung spectra with the y-axis in units of $E_{\gamma} \times$ Rate $<N>$. The $1 / E_{\gamma}$ profiles in Figure 12 now yield flat distributions. 


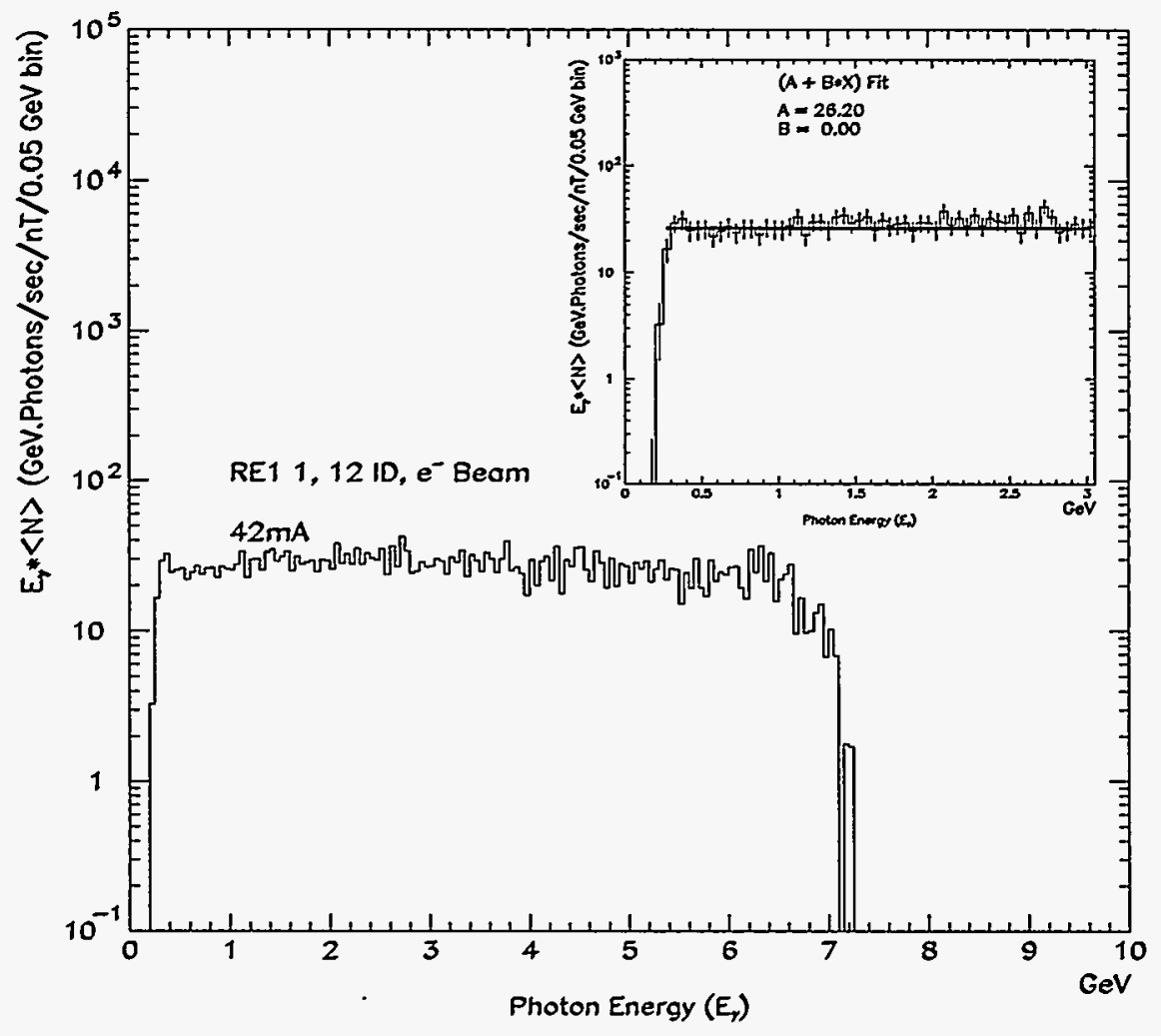

Figure 18: Dead-time corrected bremsstrahlung spectrum with the y-axis in units of $\mathrm{E}_{\gamma} \times$ Rate $<\mathrm{N}>$ fit with a straight line yielding the fit parameters $\mathrm{A}$ and $\mathrm{B}$ (inset).

estimate the total bremsstrahlung energy unaccounted for in the analysis, due to the preset threshold. This low energy correction is evidently a function of the beam current of interest. The fraction of the energy lost due to the threshold cutoff, calculated by taking the ratio of observed total energy in $\mathrm{GeV} / \mathrm{sec} / \mathrm{nT}$ from Table 3 to the corrected actual energy (obtained by adding the low energy correction from the fit procedure described above to the observed energy in Table 3 is found to be a constant for all the beam currents and runs. This fraction, calculated for a few beam currents of each of the runs, is tabulated in Table 4. It can be seen from the table that, irrespective of the beam current, approximately $2.8 \pm 0.1 \%$ of energy is being lost in the discriminator threshold 
Table 4: Low energy correction evaluated for a few typical beam currents.

\begin{tabular}{|c|c|c|c|c|c|}
\hline $\begin{array}{c}\text { RE1 \# } \\
\text { Beamline } \\
\text { and } \\
\text { Beam Type }\end{array}$ & $\begin{array}{c}\text { Beam } \\
\text { Current } \\
\\
I_{b} \\
(\mathrm{~mA})\end{array}$ & $\begin{array}{c}\text { Observed } \\
\text { Energy }^{\dagger} \\
E_{\text {uncorr }} \\
(\mathrm{GeV} / \mathrm{sec} / \mathrm{nT})\end{array}$ & $\begin{array}{c}\text { Correction } \\
\text { from Fit } \\
\\
\quad \Delta E \\
(\mathrm{GeV} / \mathrm{sec} / \mathrm{nT})\end{array}$ & $\begin{array}{c}\text { Corrected } \\
\text { Energy } \\
E_{\text {corr }}=E_{\text {uncorr }}+\Delta E \\
(\mathrm{GeV} / \mathrm{sec} / \mathrm{nT})\end{array}$ & $\begin{array}{c}\text { Fraction of } \\
\text { Energy Lost } \\
\left(1.0-\frac{E_{\text {uncorr }}}{E_{\text {corr }}}\right) \times 100 \%\end{array}$ \\
\hline 1,12 ID & 80.86 & 7,335 & 220 & 7,555 & 2.91 \\
\hline$e^{-}$ & 88.61 & 7,784 & 233 & 8,017 & 2.90 \\
\hline $2,12 \mathrm{ID}$ & $\overline{59.68}$ & 8,326 & 250 & 8,576 & 2.91 \\
\hline$e^{-}$ & 78.64 & 10,642 & 317 & 10,959 & 2.89 \\
\hline 3,10 ID & 61.65 & 3,312 & 99 & 3,411 & 2.91 \\
\hline$e^{+}$ & 78.25 & 4,894 & 145 & 5,039 & 2.88 \\
\hline $4,13 \mathrm{ID}$ & 62.35 & 15,392 & 458 & 15,580 & 2.89 \\
\hline$e^{t}$ & 77.48 & 22,829 & 678 & 23,507 & 2.88 \\
\hline
\end{tabular}

$\dagger \pm 0.4 \%$ statistical error.

setup at the low energy region. The total energy is corrected for the threshold cutoff using this fraction.

\subsection{Corrected Results for Bremsstrahlung Energy}

Table 5 gives the corrected bremsstrahlung energy results, normalized to vacuum and storage ring beam current. The results are corrected for the dead-time losses (a maximum of $3 \%$, for the largest beam current), longitudinal leakage ( $4.3 \pm 0.1 \%$ ), and threshold cutoff effects $(2.8 \pm 0.1 \%)$.

The normalized bremsstrahlung energy, as given in Table 5 , show consistent values within each individual run, but they vary from run to run and sector to sector. The results from Table 5 are also shown plotted in Figure 19 and Figure 20. Both these figures show the bremsstrahlung energy, normalized to the beam current, as a function of the storage ring vacuum for different experimental runs. Figure 19 represents data from run numbers 1 (12-ID), 2 (12-ID), and 3 (10-ID), showing straight line fits of the 
Table 5: Corrected bremsstrahlung energy results from the 15.38 meter particle beam straight path.

\begin{tabular}{|c|c|c|c|c|c|}
\hline $\begin{array}{c}\text { RE1 \# } \\
\text { Beamline } \\
\text { and } \\
\text { Beam Type }\end{array}$ & $\begin{array}{c}\text { Beam } \\
\text { Current } \\
\\
I_{b} \\
(\mathrm{~mA})\end{array}$ & $\begin{array}{c}\text { Observed } \\
\text { Energy } \\
\text { (Deadtime corrected) } \\
E_{\text {uncorr }} \\
(\mathrm{GeV} / \mathrm{sec} / \mathrm{nT})\end{array}$ & $\begin{array}{c}\text { Energy } \\
\text { Correction } \\
\text { (Leak \& Thrsh.) } \\
\Delta E \\
(\mathrm{GeV} / \mathrm{sec} / \mathrm{nT})\end{array}$ & $\begin{array}{c}\text { Corrected } \\
\text { Brems. Energy } \\
E_{\text {corr }}=E_{\text {uncorr }}+\Delta E \\
(\mathrm{GeV} / \mathrm{sec} / \mathrm{nT})\end{array}$ & $\begin{array}{c}\text { Normalized } \\
\text { Corrected } \\
\text { Brems. Energy } \\
E_{\text {corr }} / I_{b} \\
(\mathrm{GeV} / \mathrm{sec} / \mathrm{nT} / \mathrm{mA})\end{array}$ \\
\hline & 41.90 & 3,592 & 277 & 3,869 & 92 \\
\hline $1,12 \mathrm{ID}$ & 80.86 & 7,335 & 567 & 7,902 & 98 \\
\hline \multirow[t]{4}{*}{$e^{-}$} & 88.61 & 7,784 & 601 & 8,385 & 95 \\
\hline & 96.70 & 8,155 & 630 & 8,785 & 91 \\
\hline & 59.68 & 8,326 & 643 & 8,969 & 150 \\
\hline & 64.71 & 9,080 & 701 & 9,781 & 151 \\
\hline $2,12 \mathrm{ID}$ & 71.82 & 10,225 & 790 & 11,015 & 153 \\
\hline \multirow[t]{4}{*}{$e^{-}$} & 78.64 & 10,642 & 822 & 11,464 & 146 \\
\hline & 87.77 & 11,085 & 856 & 11,941 & 136 \\
\hline & 54.81 & 2,945 & 227 & 3,172 & 58 \\
\hline & 56.92 & 3,036 & 234 & 3,270 & 57 \\
\hline $3,10 \mathrm{ID}$ & 61.65 & 3,312 & 256 & 3,568 & 58 \\
\hline \multirow[t]{4}{*}{$e^{+}$} & 69.34 & 4,047 & 313 & 4,360 & 63 \\
\hline & 78.25 & 4,894 & 378 & 5,272 & 67 \\
\hline & 62.35 & 15,392 & 1,189 & 16,581 & 266 \\
\hline & 72.37 & 17,381 & 1,342 & 18,723 & 259 \\
\hline $4,13 \mathrm{ID}$ & 77.48 & 22,829 & 1,763 & 24,592 & 317 \\
\hline \multirow[t]{2}{*}{$e^{t}$} & 85.32 & 22,571 & 1,743 & 24,314 & 285 \\
\hline & 90.15 & 30,921 & 2,388 & 33,309 & 369 \\
\hline
\end{tabular}

† The statistical error on this corrected bremsstrahlung energy is $\pm 0.45 \%$. 


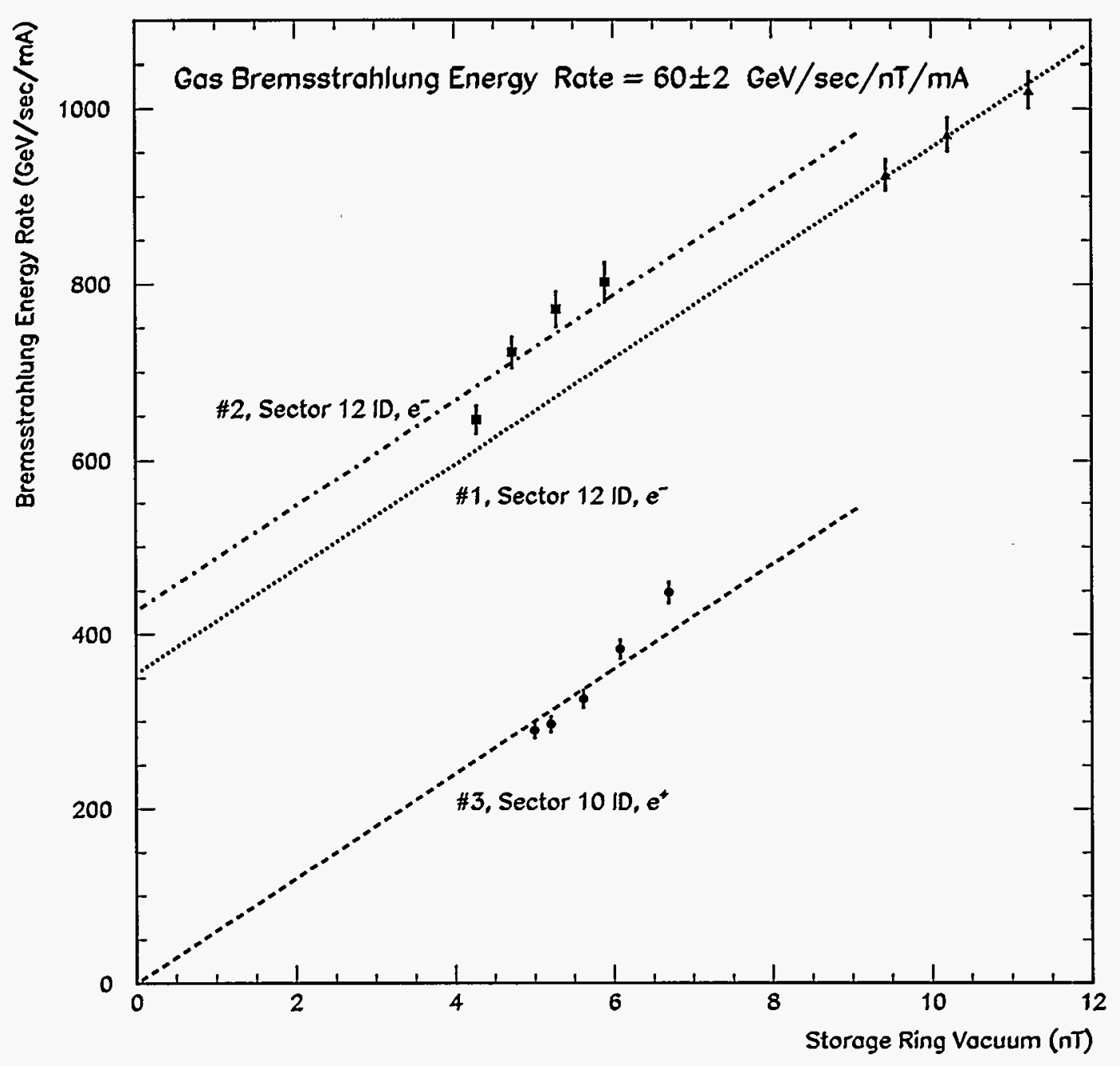

Figure 19: Bremsstrahlung energy, normalized to the beam current, as a function of the storage ring vacuum for the experimental runs conducted at sectors 10ID and 12-ID. The slopes of the fitted straight lines give the gas bremsstrahlung energy rate, the value of which is given at the top. The y-intercepts represent the non-gas bremsstrahlung energy rate from these beamlines. 


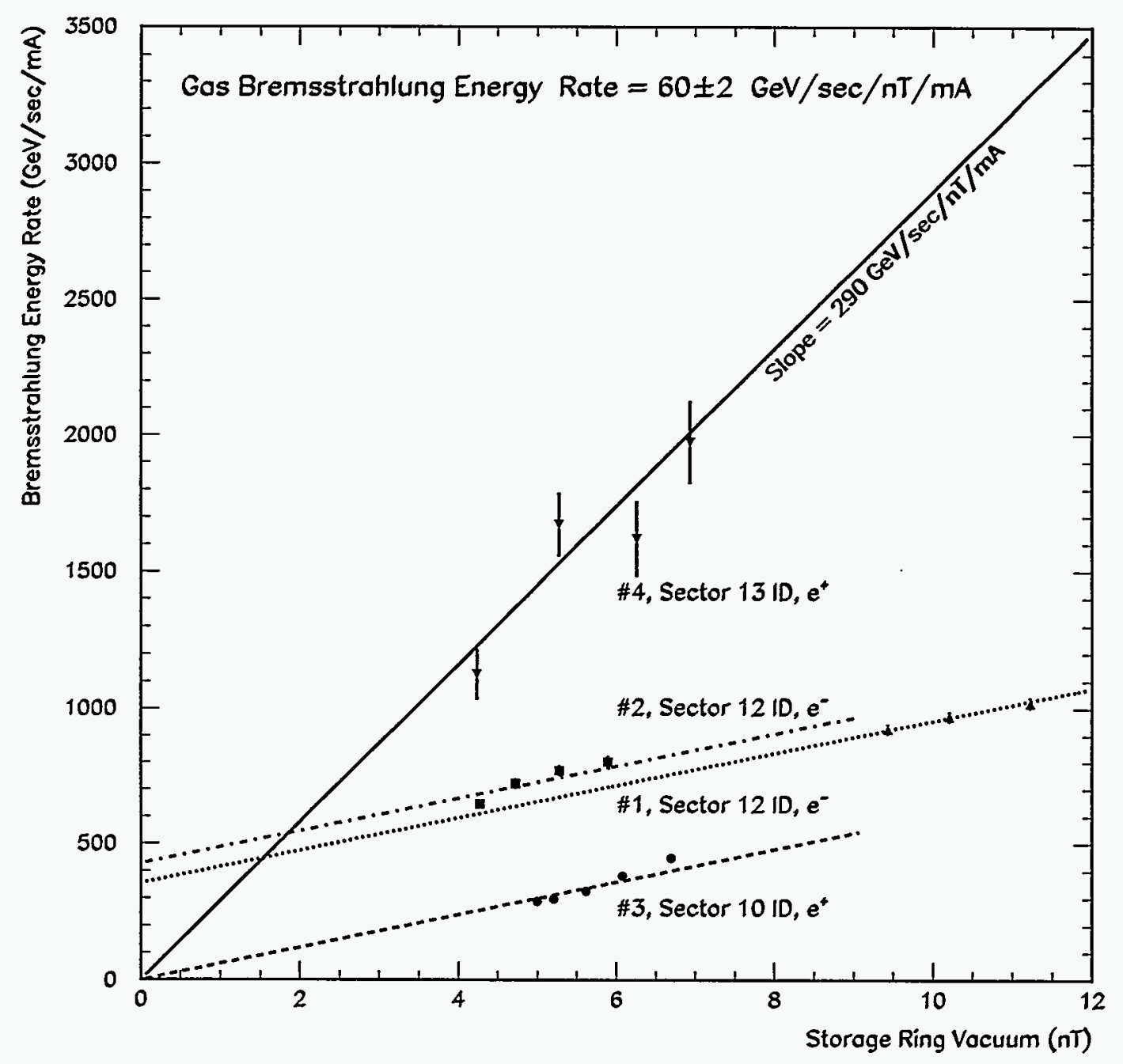

Figure 20: Data from the run conducted at sector 13-ID along with the other three data sets shown in Figure 18. The gas bremsstrahlung energy rate from 13-ID is approximately a factor of four higher compared to those from sectors 10-ID and 12-ID, consistent with a variation in the effective $\mathrm{Z}$ of the storage ring residual gas due to vacuum leak. 
form $y=m x+c, \mathrm{~m}$ being the slope and $\mathrm{c}$ being the $\mathrm{y}$-intercept. The errors shown are the measure of the deviation of each data point from the mean value as obtained from a least square fit. The slope of these fitted straight lines gives the gas bremsstrahlung energy rate from the storage ring straight path of $15.38 \mathrm{~m}$, normalized to both the vacuum and beam current. The y-intercepts of these straight lines give the bremsstrahlung energy rates from the storage ring straight path of $15.38 \mathrm{~m}$, when the vacuum of the storage ring is zero $n$ Torr. This is the contribution to the total bremsstrahlung energy rate by non-gas particle interactions in the storage ring straight path, which differ from sector to sector. The gas bremsstrahlung energy rate is calculated as $60 \pm 2 \mathrm{Gev} / \mathrm{sec} / \mathrm{nT} / \mathrm{mA}$ from Figure 19. This value is consistent for the data from run \#1 and \#2, both conducted at 12-ID, and also for the data from run \#3 conducted at 10-ID. However, as shown in Figure 20, the data from run \#4 obtained from 13-ID show a corresponding gas bremsstrahlung energy rate that is approximately a factor of four higher than those obtained from the other three data sets. This observation is consistent with a vacuum leak detected in this sector during the data collection. As mentioned earlier in section 4.3.3, presence of vacuum leaks in a sector can cause a change in the effective $Z$ of the residual gas in the straight path, giving rise to three to four times higher $\mathrm{Z}^{2}$ values than what is expected under normal operating conditions. This correspondingly enhances the gas bremsstrahlung production rate from the sector. It is also observed that, in the beam current range for which the measurements were conducted, the bremsstrahlung energy rate for a given vacuum is proportional to the current. As evident from Figure 20 , gas bremsstrahlung energy rate did not show any difference between the electron and positron runs. Thus the variations in the normalized bremsstrahlung energy rate seen in Table 5 can be attributed to the differences in the non-gas bremsstrahlung contribution from sector to sector, and to the enhanced gas bremsstrahlung production rate due to presence of vacuum leaks.

\subsection{Bremsstrahlung Dose Estimations}

When radiation is incident upon the human body, the resulting biological effects are complicated. The energy of incident radiation absorbed per unit mass, known as absorbed 
dose $D_{a b}$, is the basic physical quantity that can be associated with biological damage. But since the damage produced is also related to the type of radiation (equal amount of absorbed doses of different types of radiation produce different degrees of biological effect), another useful quantity, dose equivalent $\mathrm{D}_{e q}$, is commonly used in radiation protection applications. Thus the relative radiation hazard depends not only on the physical dose $\mathrm{D}_{a b}$, but also on other factors. $\mathrm{D}_{e q}$ is defined as the product of $\mathrm{D}_{a b}$ and $\mathrm{Q}$, where $\mathrm{Q}$ is the quality factor and is equal to 1 for electrons and photons. $D_{e q}$ therefore reflects the effectiveness of different radiation types numerically and establishes a common scale that can be used for all ionizing radiation to compare their relative hazards. In SI units, $\mathrm{D}_{e q}$ is expressed in sievert (Sv), where $1 \mathrm{~Sv}=1 \mathrm{~J} / \mathrm{kg}$.

The measured bremsstrahlung rate spectra, as shown in Figure 14, can be converted to corresponding fluence spectra by dividing them by the cross sectional area of interest. Then a set of fluence to dose equivalent conversion factors ( $F_{\text {conv }}$ in units of Sv.cm ${ }^{2}$ ) is utilized to transform the measured fluence to dose equivalent. For general radiation protection applications, it is often a common practice to associate the biological risk with the peak value of the dose equivalent distribution in the human body. This method will therefore prevent underestimating the associated risk factors. In order to properly assess this peak $\mathrm{D}_{e q}$ value from the measured fluence spectrum, its nature with respect to the depth of the material through which the radiation is passing (in the human body) has to be understood as a function of the incident energy (known as the dose equivalent vs depth curves). Since $\mathrm{F}_{\text {conv }}$ represents the dose equivalent per unit fluence, a study of $\mathrm{F}_{\text {conv }}$ vs depth curves as a function of incident energy will provide the same required information as the $\mathrm{D}_{e q}$ vs depth curves. Such a study has already been conducted and published [19] using the EGS3 [20] Monte Carlo electron-photon transport simulation code. The study was performed for broad parallel beams of mono-energetic electrons, positrons, and photons incident on a 30-cm-thick slab of ICRU four-element tissue, which normally is approximated as a human torso thickness. A summary of their $F_{\text {conv }}$ vs depth results for a few incident photon energies is shown in Figure 21. The peak or maximum of the $\mathrm{F}_{\text {conv }}$ curve, and hence that of $\mathrm{D}_{\text {eq }}$, moves from just under the ICRU4 surface (lower depth values on the $\mathrm{x}$-axis) to deep within, and eventually to the outside $(>30 \mathrm{~cm})$ as the $\mathrm{E}_{\gamma}^{\text {inc }}$ increases from $20 \mathrm{MeV}$ to $5 \mathrm{GeV}$. The peak value of $\mathrm{F}_{\text {conv }}$ itself is successively 


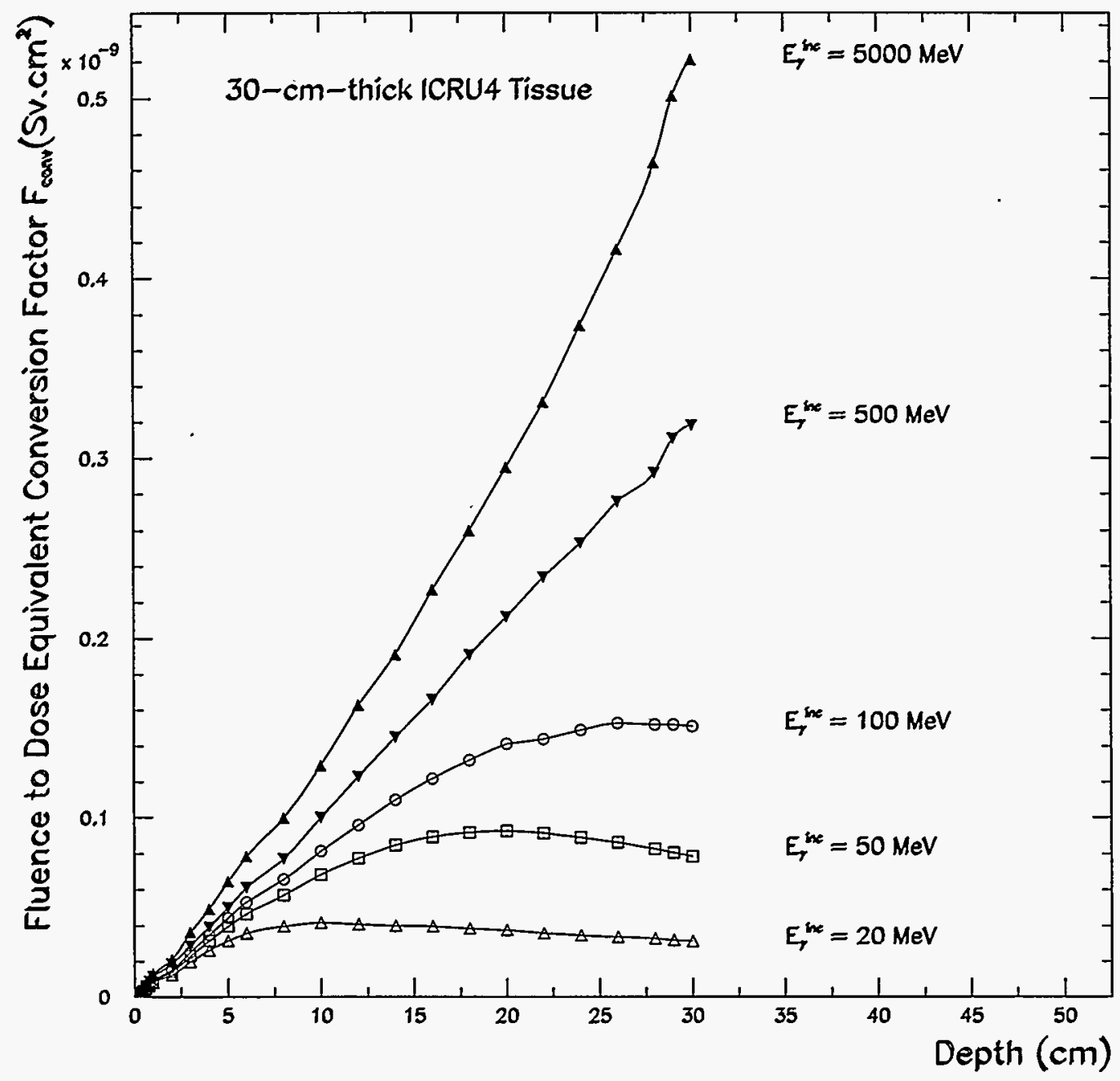

Figure 21: The fluence-to-dose-equivalent conversion factors $\left(\mathrm{Sv} . \mathrm{cm}^{2}\right)$ vs depth curves for varying incident photon energies $\left(\mathrm{E}_{\gamma}^{\text {inc }} \mathrm{GeV}\right)$. The results are obtained from calculations conducted by D.W.O. Rogers [19]. 
higher for each of the given incoming energies. Approximately above $100 \mathrm{MeV}$, the dose profile peaks outside the $30 \mathrm{~cm}$ thick ICRU4 tissue slab. For incident energies beyond $100 \mathrm{MeV}$ or so, because the peak will be situated outside the ICRU4 region, the largest $\mathrm{F}_{\text {conv }}$ value within the 30 -cm-thick region, $\mathrm{F}_{c o n v}^{L R G}$, has been used in this analysis. The $\mathrm{F}_{\text {conv }}^{L R G}$ values corresponding to the bremsstrahlung energy range are shown in Figure 22.

The bremsstrahlung rate profiles in Figure 14 are converted into their respective fluence spectra by dividing them by a cross sectional area that corresponds to the average bremsstrahlung beam size at the detector location. This cross sectional area is determined from the average bremsstrahlung emission angle [7] at the source point, 26 $m$ upstream. At $7 \mathrm{GeV}$ APS electron energy, an average emission angle value of 73 $\mu \mathrm{rad}$ can be estimated. The average bremsstrahlung beam cross sectional area, $26 \mathrm{~m}$ downstream with this characteristic angle of emission, is then approximately $0.113 \mathrm{~cm}^{2}$. The conversion factor curve in Figure 22 is then used in conjunction with the converted fluence spectra to obtain corresponding maximum dose equivalent spectra. The correction for the dose from the events missed due to the threshold setup has also been investigated. It was shown that approximately $2.8 \%$ of the total energy is lost in the threshold cutoff region of $0-150 \mathrm{MeV}$ (section 4.4.3). Since dose is defined as the energy absorbed per unit mass, its contribution in the threshold cutoff region will also be $2.8 \%$ of the total dose which is small enough to be ignored for all practical purposes. The maximum dose equivalent values corresponding to a few typical results in Table 5, calculated by summing the events under the dose equivalent spectra along the entire energy range, are given in Table 6 in units of $\mathrm{Sv} / \mathrm{hr} / \mathrm{nT} / \mathrm{mA}$. From this table, it can be seen that the dose equivalent rate that closely represents the gas bremsstrahlung is $4.2 \times 10^{-4} \mathrm{~Sv} / \mathrm{hr} / \mathrm{nT} / \mathrm{mA}$ which corresponds to $0.13 \mathrm{~Sv} / \mathrm{hr} / \mathrm{nT}$ for a beam current of 300 $\mathrm{mA}$. The maximum bremsstrahlung energy rate measured at an ID beamline in this experiment is $369 \mathrm{GeV} / \mathrm{sec} / \mathrm{nT} / \mathrm{mA}$ (see Table 5) which yields a dose equivalent rate of $2.18 \times 10^{-3} \mathrm{~Sv} / \mathrm{hr} / \mathrm{nT} / \mathrm{mA}$. At this rate, the dose equivalent value is $0.65 \mathrm{~Sv} / \mathrm{hr} / \mathrm{nT}$ for a beam current of $300 \mathrm{~mA}$. 


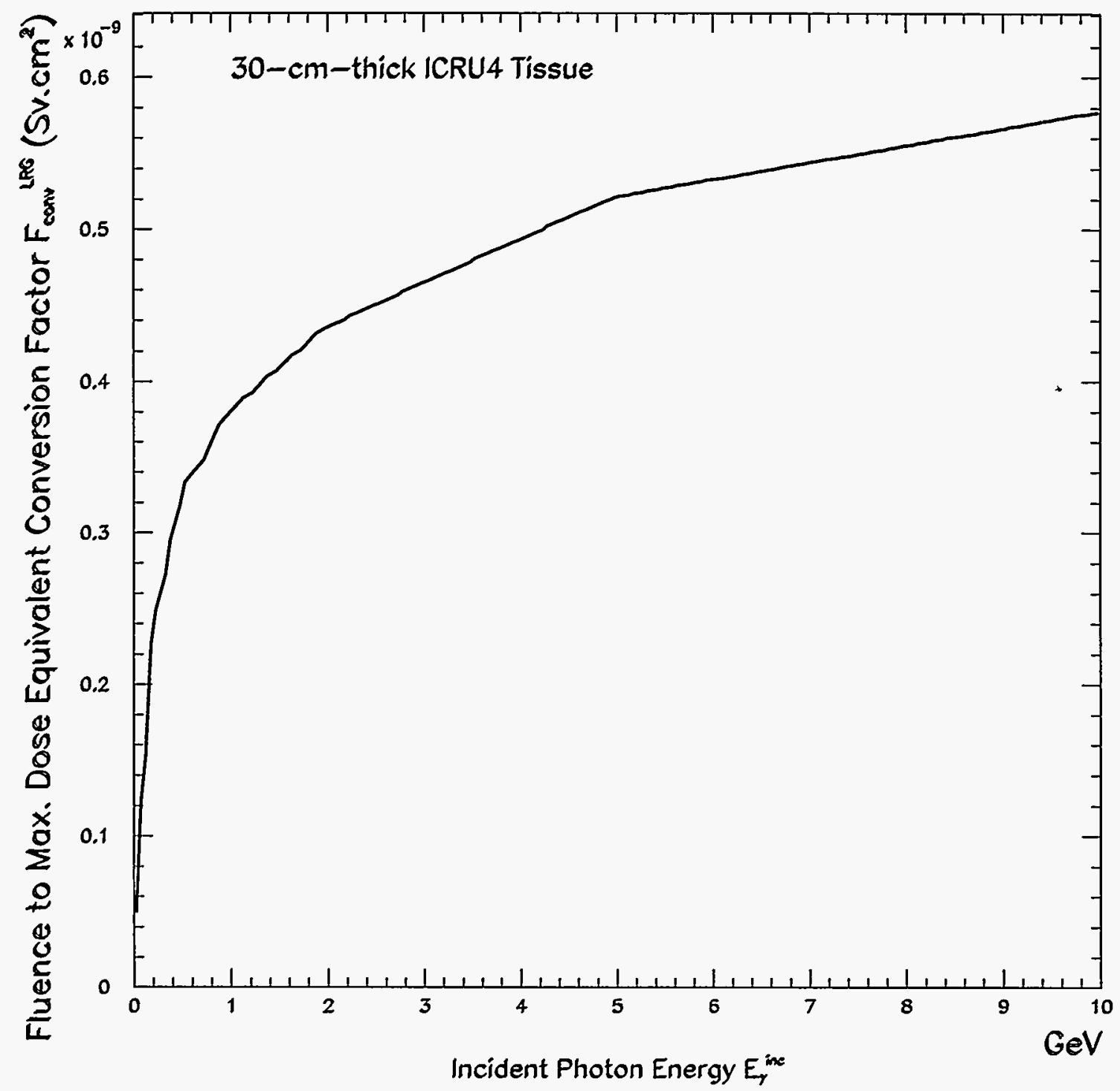

Figure 22: The calculated largest fluence-to-dose-equivalent conversion factors within the $30 \mathrm{~cm}$ ICRU4 tissue thickness as a function of incident bremsstrahlung energy. 
Table 6: Normalized maximum dose equivalent values corresponding to the bremsstrahlung energy radiated from the $15.38 \mathrm{~m}$ of particle beam straight path. Only one typical value per run is shown.

\begin{tabular}{|c|c|c|c|c|}
\hline \hline $\begin{array}{c}\text { RE1 \# } \\
\begin{array}{c}\text { Beamline } \\
\text { and } \\
\text { Beam Type }\end{array}\end{array}$ & $\begin{array}{c}\text { Beam } \\
\text { Current } \\
I_{b} \\
(\mathrm{~mA})\end{array}$ & $\begin{array}{c}\text { Estimated } \\
\text { Vacuum } \\
(\mathrm{nT})\end{array}$ & $\begin{array}{c}\text { Normalized } \\
\text { Corrected } \\
\text { Brems. Energy } \\
E_{\gamma} \\
(\mathrm{GeV} / \mathrm{sec} / \mathrm{nT} / \mathrm{mA})\end{array}$ & $\begin{array}{c}\text { Maximum } \\
\text { Dose } \\
\text { Equivalent } \\
D_{e q} \\
(\mathrm{~Sv} / \mathrm{hr} / \mathrm{nT} / \mathrm{mA})\end{array}$ \\
\hline $\mathbf{1 , 1 2} \mathrm{ID}, e^{-}$ & 80.86 & 9.43 & 98 & $5.74 \times 10^{-4}$ \\
\hline $\mathbf{2 , 1 2} \mathbf{I D}, e^{-}$ & 78.64 & 5.28 & 146 & $9.13 \times 10^{-4}$ \\
\hline $\mathbf{3 , 1 0} \mathbf{I D}, e^{+}$ & 78.25 & 6.69 & 67 & $4.20 \times 10^{-4}$ \\
\hline $\mathbf{4 , 1 3} \mathbf{I D}, e^{+}$ & 90.15 & 8.49 & 369 & $2.18 \times 10^{-3}$ \\
\hline \hline
\end{tabular}




\section{Chapter 5}

\section{Conclusions}

The lead glass calorimeter, by virtue of its sensitivity and fast response, is found to be a very effective detector for the accurate measurement of bremsstrahlung from electron storage rings. This calorimeter can be calibrated using the maximum energy of the observed bremsstrahlung spectrum, which is equal to the energy of the particle beam in the storage ring. The gas bremsstrahlung rate from the APS undulator beamline straight path of $15.38 \mathrm{~m}$ is measured as $60 \pm 2 \mathrm{GeV} / \mathrm{sec} / \mathrm{nT} / \mathrm{mA}$. This corresponds to a maximum dose equivalent rate of approximately $4.2 \times 10^{-4} \mathrm{~Sv} / \mathrm{hr} / \mathrm{nT} / \mathrm{mA}$. The analysis shows that there is a significant bremsstrahlung contribution from sources other than the residual gas molecules in the beam straight path of the storage ring. The maximum bremsstrahlung energy rate measured in a sector during this experiment is 369 $\mathrm{GeV} / \mathrm{sec} / \mathrm{nT} / \mathrm{mA}$, which corresponds to a maximum dose equivalent value of approximately $2.18 \times 10^{-3} \mathrm{~Sv} / \mathrm{hr} / \mathrm{nT} / \mathrm{mA}$. The shielding provided for the first optical enclosures and the white beam stations are adequate to contain this bremsstrahlung dose rate. In the beam current range for which the measurements were conducted, the bremsstrahlung energy rate for a given storage ring vacuum is found to be proportional to the current. We did not observe any difference in the measured gas bremsstrahlung energy rate between the positron and electron runs. A measurement of the residual gas composition inside the storage ring straight path shows $\mathrm{CO}, \mathrm{CO}_{2}$, and $\mathrm{H}_{2}$ to be the major constituting components. 


\section{Bibliography}

[1] H.A. Bethe and W. Heitler. Proc. Roy. Soc., A146, 84 (1934).

[2] W. Heitler. Quantum Theory of Radiation. Oxford University Press., London, (1944).

[3] H.W. Koch and J.W. Motz. Bremsstrahlung Cross-Section Formulas and Related Data. Rev. Mod. Phys., 31, 920 (1959).

[4] B. Rossi. High Energy Particles. Prentice Hall, Englewood Cliffs, NJ (1952).

[5] G.K. Shenoy, P.J. Viccaro, and D.M. Mills. Characteristics of the 7-GeV Advanced Photon Source: A Guide for Users. ANL-88-9, Argonne National Laboratory, February 1988.

[6] H.J. Moe. Advanced Photon Source: Radiological Design Considerations. APS-LS141, Argonne National Laboratory, July 1991.

[7] L.I Schiff. Chapter 20, Sec. 1. Phys. Rev., 70, (1946).

[8] G. Bathow et al. Measurements on $6.3 \mathrm{GeV}$ Electromagnetic Cascades and Cascade Produced Neutrons. Nucl. Phys., B2, 669 (1967).

[9] S. Ban et al. Estimation of Absorbed Dose due to Gas Bremsstrahlung from Electron Storage Rings. Health Phys., 57, 407 (1989).

[10] A. Rindi. Gas Bremsstrahlung from Electron Storage Rings. Health Phys., 42, 187 (1982). 
[11] A. Esposito and M. Pelliccioni. Gas Bremsstrahlung Production in the ADONE Electron Storage Ring. LNF 86/23., INFN Report, (1986).

[12] A. Esposito and M. Pelliccioni. Gas Bremsstrahlung Production in the ADONE Electron Storage Ring. Proc. Health Phys. Soc., Reno CONF-8602106, 495 (1987).

[13] G. Tromba and A. Rindi. Gas Bremsstrahlung, A Monte Carlo Evaluation. Nucl. Instr. Methods., A292, 700 (1990).

[14] A. Ferrari et al. Estimation of Fluence Rate and Dose Rate due to Gas Bremsstrahlung from Electron Storage Rings. Nucl. Instr. Meth., B83, 518 (1993).

[15] J.C Frank. LURE EP 88-01, (1988).

[16] J. Andruszkow et al. Calibration Procedure of the Calorimeters of the ZEUS Luminosity Monitor. ZEUS Note 94-071, HERA, Hamburg, (1994).

[17] W.R. Nelson et al. EGS4 Code System SLAC-265, SLAC, Stanford, (1985).

[18] P.K. Job et al. Initial Bremsstrahlung Measurements in an APS Insertion Device Beamline. ANL, Internal Memo (1995).

[19] D.W.O. Rogers. Fluence to Dose Equivalent Conversion Factors Calculated with EGS3 for Electrons from $100 \mathrm{keV}$ to $20 \mathrm{GeV}$ and Photons from $11 \mathrm{keV}$ to $20 \mathrm{GeV}$. Health Phys., 46, 891 (1984).

[20] R.L. Ford and W.R. Nelson. The EGS Code System SLAC-210, SLAC, Stanford, (1978). 